\title{
Black funnels and droplets in thermal equilibrium
}

\author{
Marco M. Caldarelli, ${ }^{a, b}$ Óscar J.C. Dias, ${ }^{c}$ Ricardo Monteiro $^{d}$ and Jorge E. Santos ${ }^{e}$ \\ ${ }^{a}$ Laboratoire de Physique Théorique, Univ. Paris-Sud, CNRS UMR 8627, \\ F-91405 Orsay, France \\ ${ }^{b}$ Centre de Physique Théorique, Ecole Polytechnique, CNRS UMR 7644, \\ F-91128 Palaiseau, France \\ ${ }^{c}$ DAMTP, Centre for Mathematical Sciences, University of Cambridge, \\ Wilberforce Road, Cambridge CB3 0WA, United Kingdom \\ ${ }^{d}$ The Niels Bohr International Academy, The Niels Bohr Institute, \\ Blegdamsvej 17, DK-2100 Copenhagen, Denmark \\ e Department of Physics, UCSB, \\ Santa Barbara, CA 93106, U.S.A. \\ E-mail: marco.caldarelli@th.u-psud.fr, 0.Dias@damtp.cam.ac.uk, \\ monteiro@nbi.dk, jss55@physics.ucsb.edu
}

ABSTRACT: It has recently been proposed that the strong coupling behaviour of quantum field theories on a non-dynamical black hole background can be described, in the context of the AdS/CFT correspondence, by a competition between two gravity duals: a black funnel and a black droplet. We present here thermal equilibrium solutions which represent such spacetimes, providing the first example where the thermal competition between the gravity duals can be studied. The solutions correspond to a special family of charged AdS C-metrics. We compute the corresponding Euclidean actions and find that the black funnel always dominates the canonical ensemble in our example, meaning that the field theory does not undergo a phase transition.

KeYwORDS: AdS-CFT Correspondence, Black Holes

ARXIV EPRINT: 1102.4337 


\section{Contents}

1 Introduction 1

2 Black droplets and funnels in thermal equilibrium 3

2.1 The charged AdS C-metric 3

2.2 A special family of lukewarm AdS C-metrics 5

$\begin{array}{lll}2.3 & \text { Black droplets and funnels in thermal equilibrium } & 7\end{array}$

$\begin{array}{lll}3 & \text { Free energy of the black droplets and funnels } & 13\end{array}$

4 Holographic interpretation of black droplets and funnels 21

A The charged AdS C-metric: black droplets and black funnels 25

$\begin{array}{ll}\text { B Configurations in thermal equilibrium } & 30\end{array}$

\section{Introduction}

Black holes are simple and highly symmetric solutions of general relativity, whose classical behavior is fairly well understood. In a more general framework, they are located at the interface between classical and quantum realms, and for this reason they stand out in the quest for a quantum theory of gravity. The most striking and well-known effect due to quantum theory is that black holes - classically perfect absorbers - evaporate through the emission of thermal Hawking radiation $[1,2]$.

Unfortunately, the calculations leading to the Hawking radiation are quite complicated; they can be carried out in general only for free fields propagating on the curved background of the black hole, and weakly interacting fields in perturbation theory, see for example [3, 4]. Very little is known on the other hand when the fields interact strongly, and the best one can do is to define a Hartle-Hawking state using a Euclidean path integral periodic in imaginary time.

A new road to explore these phenomena has been opened in a series of articles by Hubeny, Marolf and Rangamani [5-7] using the power of the AdS/CFT correspondence [810], that maps strongly coupled quantum field theories to the classical gravitational dynamics in an higher dimensional AdS spacetime. The state describing the quantum field theory at strong coupling propagating on a non-dynamical manifold $\mathcal{M}$ is holographically described by an AdS supergravity solution whose timelike boundary is conformal to $\mathcal{M}$. Ideally, one would like to find the bulk geometry whose boundary is conformal to the Schwarzschild spacetime. Such a solution would describe Hawking radiation at strong coupling. It is however challenging to obtain such a solution without resorting to numerical 
a)

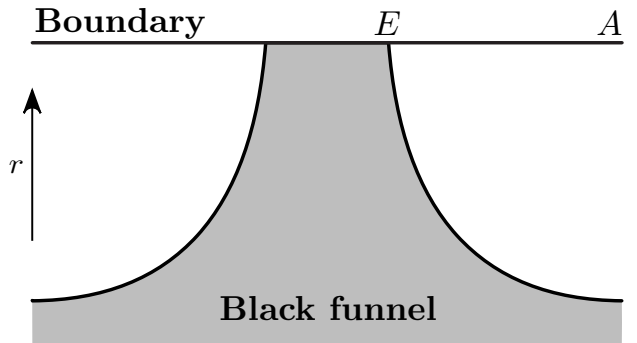

b)

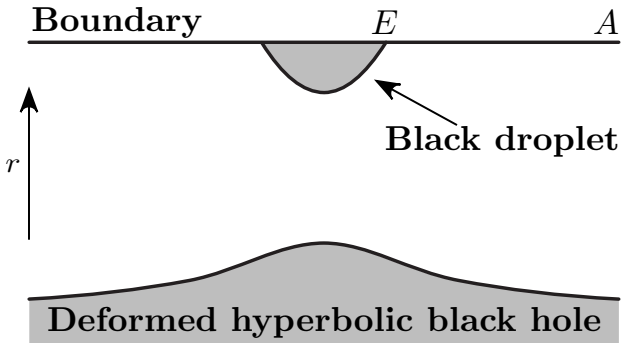

Figure 1. a) Schematic spatial representation of a black funnel. It has a throat that starts at the boundary black hole, and then extends into the bulk where, at the funnel "shoulders", it approaches the geometry of a hyperbolic black hole. Coordinate $r$ is the holographic radial coordinate. The holographic boundary is at $r \rightarrow \infty$. Point $E$ signals the horizon of the boundary black hole. Point $A$ describes the asymptotic region of the holographic boundary. b) Black droplet system with two disconnected horizons. One of them, that joins the horizon of the boundary black hole, describes a black droplet. The other one, deep in the bulk, describes a deformed hyperbolic black hole that approaches the geometry of a hyperbolic black hole for large values of the radial boundary coordinate.

work, and for this reason simplified models were initially analyzed: in [5, 6], Hubey, Marolf and Rangamani studied three-dimensional black holes appearing on the conformal boundary of the AdS C-metric, while in [7] the problem was approached by the same authors for AdS black hole backgrounds.

Traditionally, the (AdS) C-metric is taken to describe a pair of uniformly accelerated black holes, the acceleration being driven by a string that pulls the black hole (see, e.g., [1114]). The authors of [5] showed that a different interpretation is possible, for which the conformal timelike boundary is a spacetime containing a black hole, whose event horizon is continued in the bulk of the solution as shown in figure 1. The event horizon of the bulk solution can be singly connected, and asymptote to the event horizon of a black brane (figure 1.a): in that case, the solution describes a black funnel. Alternatively, the event horizon of the boundary black hole can close off in the bulk, forming a black droplet floating over a planar black brane that lives deep in the bulk of the dual spacetime, as shown in figure 1.b. These two situations arise in two different regimes of the Hawking radiation, the former when the boundary black hole couples strongly with the field theory plasma, while the latter, due to the lack of connectivity of the two horizons, describes a situation where the boundary black hole polarizes the vacuum near the horizon, but couples only weakly to the field theory plasma dual to the black brane.

So, for each boundary black hole there are two possible bulk solutions that have the same conformal timelike boundary, one describing a black funnel, and the other a black droplet. Both these solutions form gravitational instantons that contribute to the full Euclidean action of the boundary black hole and compete to dominate the thermodynamical ensemble. In [5], a phase transition was conjectured between funnel dominated ensembles and black droplet dominated ensembles as the size $R_{\mathrm{bdy}}$ of the asymptotically flat boundary black hole and the the distance $R_{\mathrm{S}}$ between the "shoulder" of the planar black brane or black funnel and the holographic boundary are tuned. In the neutral system of [5] the distance 
$R_{\mathrm{S}}$ scales with the inverse of the temperature, $R_{\mathrm{S}} \sim 1 / T_{H}$. More precisely, one should expect the dynamics of small black holes with $R_{\text {bdy }} T_{H} \ll 1$ to be driven by black droplets, while black funnels should describe the physics of large, $R_{\mathrm{bdy}} T_{H} \gg 1$, asymptotically flat boundary black holes. This could however not be tested by the authors of [5], because the uncharged C-metric can never be in thermal equilibrium; the black droplet and the planar black hole have different temperatures and one cannot compare the free energies of the black funnel and the black droplet.

In this article, we want to explore these ideas further by considering a more general solution, the charged AdS C-metric [11, 13] solution of the four dimensional EinsteinMaxwell theory with negative cosmological constant. Having at our disposal one extra parameter - the charge of the black hole - we can tweak it to reach thermal equilibrium. This is seen to happen in the case of "lukewarm" black droplets [15]-[23]. However, the extra parameter is not enough to make the chemical potentials of the two event horizons of the black droplet spacetime to match. Such a match is a necessary condition for the thermodynamic equilibrium in the grand-canonical ensemble. Therefore, we shall consider only the canonical ensemble. Another important point is that, when comparing the free energies, we will work only with the magnetically charged solutions. While the charged AdS C-metric allows for electric and/or magnetic charge, the electromagnetic potential in the electric case cannot be made everywhere regular in the Euclidean section.

The paper is organised as follows. In section 2, we will find the solutions in thermal equilibrium and study their geometry. Section 3 will be devoted to the computation of the Euclidean action for the corresponding black funnel and "lukewarm" black droplet solutions. This will allow us to compare the free energies and show that there is no sign of a phase transition: the black funnels are always the dominant instanton. This can be understood by the fact that the black droplet and the planar black hole have charges of opposite sign, which has a cost in terms of greater free energy of the total system. We conclude in section 4, where we compute the expectation value of the stress tensor of the quantum fields propagating on the boundary black hole background, and we discuss the quantum field theory interpretation of the black funnels and droplets. Finally, in the appendices we first give a detailed analysis of the charged AdS C-metric parameter space (appendix A), followed by the analysis of the solutions with horizons in thermal equilibrium (appendix B) and a symmetry of the lukewarm solutions that simplifies their study.

\section{Black droplets and funnels in thermal equilibrium}

\subsection{The charged AdS C-metric}

The charged AdS C-metric [11] is a Petrov type D solution of four-dimensional EinsteinMaxwell theory in presence of a cosmological constant $\Lambda=-3 / \ell^{2}$, and has been extensively studied in recent years [12-14] in many different contexts [22]-[34]. Its line element can be written as

$$
d s^{2}=\frac{1}{\mathcal{A}^{2}(x-y)^{2}}\left(-F(y) \mathcal{A}^{2} \ell^{2} d t^{2}+\frac{d y^{2}}{F(y)}+\frac{d x^{2}}{G(x)}+G(x) d \phi^{2}\right)
$$


with fourth order polynomials $F$ and $G$ defined by

$$
G(x)=1-\kappa x^{2}-2 \mu x^{3}-\left(q_{e}^{2}+q_{m}^{2}\right) x^{4}, \quad F(y)=\frac{1}{\mathcal{A}^{2} \ell^{2}}-G(y) .
$$

The associated Maxwell potential is given by

$$
A=-q_{e} \ell y d t+\frac{q_{m}}{\mathcal{A}} x d \phi
$$

and the metric and gauge field satisfy the field equations

$$
\begin{aligned}
\nabla_{\mu} F^{\mu \nu} & =0, \\
R_{\mu \nu}-\frac{1}{2} R g_{\mu \nu}+\Lambda g_{\mu \nu} & =8 \pi \mathcal{G} T_{\mu \nu}^{\mathrm{Max}}, \quad \text { with } \quad T_{\mu \nu}^{\mathrm{Max}}=\frac{1}{4 \pi \mathcal{G}}\left(F_{\mu}{ }^{\alpha} F_{\nu \alpha}-\frac{1}{4} g_{\mu \nu} F_{\alpha \beta} F^{\alpha \beta}\right)
\end{aligned}
$$

being the Maxwell energy-momentum tensor associated with the field strength $F=d A$.

This solution is characterized by six parameters: the cosmological constant $\Lambda$, the acceleration parameter $\mathcal{A}$, the mass parameter $\mu$, the gauge charges parameters $q_{e}$ (electric) and $q_{m}$ (magnetic), and the parameter $\kappa$ which determines the topology of the black holes ( $\kappa=1,0,-1$ for horizons with spherical, planar and hyperbolic topology, respectively). The geometry is asymptotically locally anti-de Sitter (AdS), the asymptotic region being in the neighborhood of the $x=y$ hypersurface, and has curvature singularities at $y= \pm \infty$ and at $x= \pm \infty$.

If we choose appropriately the allowed range of the $(x, y)$ coordinates, the AdS Cmetric describes a regular spacetime with horizons clothing the curvature singularities. To guarantee that the geometry has the correct Lorentzian signature $(-,+,+,+)$, we must demand that the range of $x$ is such that $G(x) \geq 0$, and since $G(x)<0$ for large $x$, it follows that the coordinate $x$ is restricted to vary between two roots $x_{i}$ and $x_{j}$ of the polynomial $G(x)$. These roots signal the presence of axes of symmetry of the solution, corresponding to the rotational symmetry generated by $\partial_{\phi}$. Typically, the C-metric develops conical singularities at these poles. To avoid the conical singularity at one of the poles, say $x_{i}$, we choose the period of $\phi$ to be given by

$$
\Delta \phi=\frac{4 \pi}{\left|G^{\prime}\left(x_{i}\right)\right|},
$$

but, this leaves a conical singularity at the other pole with deficit angle given by

$$
\delta_{j}=2 \pi\left(1-\frac{G^{\prime}\left(x_{j}\right)}{\left|G^{\prime}\left(x_{i}\right)\right|}\right) .
$$

A crucial feature for us is that the asymptotic boundary conditions that one sets on the $x=y$ hypersurface can lead to two different external solutions for the field equations, one obtained in the region $y \leq x$ and the other for $y \geq x$. The range of $y$ is therefore chosen to be $y \leq x$ (or $y \geq x$ ); the upper (lower) bound $y=x$ describes the asymptotic AdS region and $y=-\infty$ (or $y=+\infty$ ) corresponds to a curvature singularity.

The roots of $F(y)$ usually describe the horizons of the C-metric solution. These can be up to four, labelled $y_{i}$ with $i=0 \ldots 3$, and ordered according to $y_{0} \leq y_{1} \leq y_{2}<y_{3}$. The 
Hawking temperature $T_{H_{i}}$ associated to the event horizons is given by their surface gravity evaluated on them, divided by $2 \pi$, i.e. $T_{H_{i}}^{2}=-\left.\left(8 \pi^{2}\right)^{-1}\left(\nabla_{\mu} \xi_{\nu}\right)\left(\nabla^{\mu} \xi^{\nu}\right)\right|_{H_{i}}$, where $\xi=\partial_{t}$ is the null generator of the Killing horizon $H_{i}$. For the C-metric, this gives

$$
T_{H_{i}}=\frac{\mathcal{A} \ell\left|F^{\prime}\left(y_{i}\right)\right|}{4 \pi} .
$$

In the standard interpretation of the C-metric as a pair of uniformly accelerated black holes, the solution parameters are usually strongly restricted to guarantee the existence of a continuous limit as the acceleration parameter goes to zero, where one recovers the AdS charged black holes. For example, for $\kappa=1$, the range of $x$ is constrained to be such that after taking the coordinate transformation $y=-1 /(\mathcal{A} r)$ and $x=\cos \theta$ and setting $\mathcal{A}=0$, one recovers the standard AdS Reissner-Nordström black hole with $-1 \leq x \leq 1$. The horizons at $y_{0}$ and $y_{1}$ are then, respectively, the inner and outer black hole horizons and $y=y_{3}$ is the acceleration horizon. One avoids the conical singularity at $x=-1$ by choosing the period of $\phi$ to be given by (2.5) and the deficit angle that is left at $x=1$ is interpreted to be sourced by a string, with mass density $\mu_{s}=\delta /(8 \pi)$ and pressure $p=-\mu_{s}$, that accelerates the black hole.

\subsection{A special family of lukewarm AdS C-metrics}

In the present study, we want to look into the C-metric from a different perspective. Instead of interpreting it as a pair of accelerated black holes we want to discuss it as a solution that describes black droplets and black funnels. The idea is to extend the analysis of $[5,6]$ to the charged case, with the advantage that the presence of the charge allows us to have solutions in thermal equilibrium. This interpretation demands imposing different requirements from the standard ones, discussed above, on the range of the solution parameters. We will do a detailed survey over the several possible C-metric solutions in section A. However, in this section, we will focus on the most interesting case for our purposes.

As motivated in the introduction, we are interested in solutions that describe a black droplet in thermal equilibrium with a deformed hyperbolic black hole in the bulk. This translates into finding families of parameters of the AdS C-metric that have:

i) two non-degenerate event horizons at the same temperature;

ii) a regular electromagnetic field;

iii) a boundary black hole geometry with a well defined asymptotic region.

As will be explained in more detail later, solutions satisfying condition iii) have a double root in the function $G(x)$ defined in (2.2). Families of solutions with these properties are described by functions $F(y)$ and $G(x)$ that have the behaviour sketched in figure 2. As for condition ii), we mean not only that the Maxwell tensor $F$ is regular, but also that the electromagnetic potential $A$ (in the Euclideanised solution) can be put in a gauge where it is explicitly regular. We will for now neglect this restriction, and will address it in section 3.

Condition i) requires that the temperatures (2.7) of the two event horizons coincide. In the charged AdS C-metric there are four families of solutions where two of the horizons have 

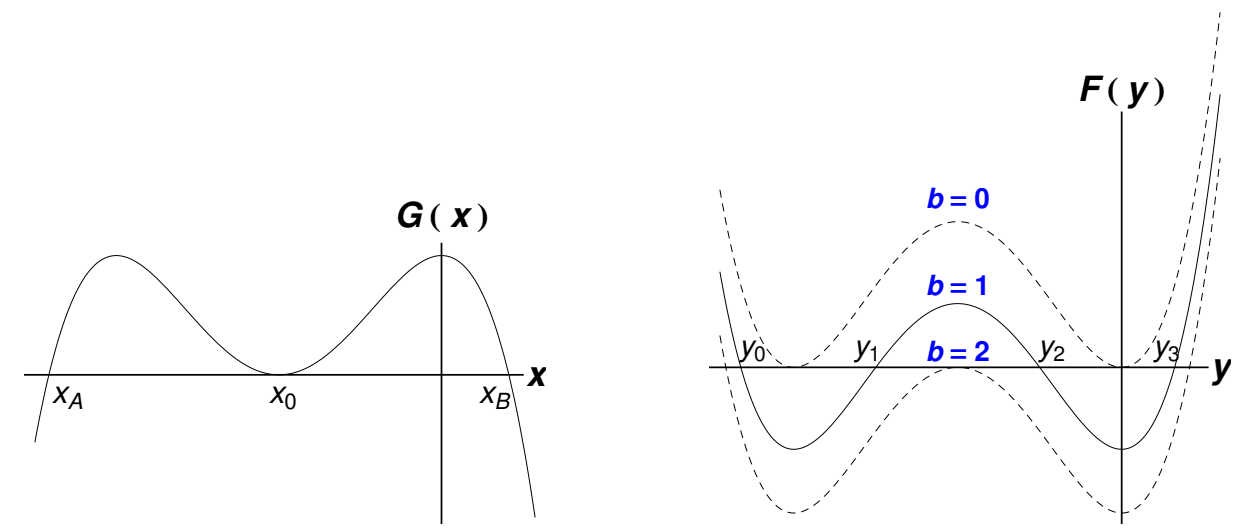

Figure 2. Functions $G(x)$ and $F(y)$ for the lukewarm charged AdS C-metric with a double zero in $G(x)$ : see (2.9). As explained in the text, the double root $x_{0}=-2$ of $G(x)$ is at an infinite proper distance from any other point with $x \neq x_{0}$. There are therefore two disjoint regions of interest where $G(x)>0: x_{A} \leq x \leq x_{0}$ and $x_{0} \leq x \leq x_{B}$, with $x_{A}=-2(\sqrt{2}+1)$ and $x_{B}=2(\sqrt{2}-1)$. On the right panel, we show how the graph of $F(y)$ gets shifted as the parameter $b$ varies in its range $0 \leq b \leq 2$. It has four zeros if $0<b<2$; two zeros if $b=0$; and three zeros if $b=2$. The horizons at $y_{1} \equiv-a=b-4$ and $y_{2} \equiv-b$ have the same temperature in all these cases.

the same temperature. In the literature, these are denoted by the Nariai, cold, ultracold and lukewarm solutions [23]. ${ }^{1}$ The former three families require zero temperature horizons and we discard them. The lukewarm solution is the only one that interests us. It describes a solution where the horizons located at $y=y_{1}$ and $y=y_{2}$ have a non-vanishing temperature. Moreover, in general these horizons do not coincide, and their temperature is finite. On the other hand, condition iii) implies that the real zeros of $G$ satisfy $x_{A}<x_{0}=x_{1}<x_{B}$ (see figure 2.a).

The general analysis of the lukewarm solutions is performed in appendix B, where it is shown that conditions i) and iii) are satisfied for a one parameter (for fixed $\ell$ and fixed $q_{e}$ or $\left.q_{m}\right)$ subfamily of the AdS C-metric, with spherical horizons $(\kappa=+1)$ and with the property that the mass parameter $\mu$ and total charge parameter $\sqrt{q_{e}^{2}+q_{m}^{2}}$ are equal. In short, this solution satisfies

$$
F\left(y_{1}\right)=F\left(y_{2}\right)=0, \quad F^{\prime}\left(y_{1}\right)=-F^{\prime}\left(y_{2}\right) ; \quad \text { and } \quad G\left(x_{0}\right)=0, \quad G^{\prime}\left(x_{0}\right)=0,
$$

where $y_{1} \equiv-a, y_{2} \equiv-b$ and $x_{0}$ are the roots displayed in figure 2 . The conditions on

\footnotetext{
${ }^{1}$ In the standard interpretation of the C-metric as a pair of accelerated black holes, the Nariai solution describes the case where the outer black hole horizon $y_{1}$ coincides with the acceleration horizon $y_{2}$; in the cold solution the inner $\left(y_{0}\right)$ and outer $\left(y_{1}\right)$ black hole horizons coincide; and the ultracold solution is the limiting case where $y_{0}=y_{1}=y_{2}$. The cold and ultracold solutions have zero temperature horizons, justifying their name. In the lukewarm solution, as the name suggests, the two horizons have the same non-vanishing temperature. This nomenclature was first introduced by Mellor and Moss [15, 16], and Romans [17] for de Sitter solutions (see also [21] for a generalization of these solutions to higher dimensions). The acceleration horizon in the C-metric solution plays in many respects a similar role as the cosmological horizon in the de Sitter black holes. Thus it is not a surprise that solutions with horizons at equal temperature share a similar structure in the two systems and that the same nomenclature is used [18]-[23].
} 
$G\left(x_{0}\right)$ and $G^{\prime}\left(x_{0}\right)$ require that $a=4-b$. Explicitly, the lukewarm family of solutions that satisfies (2.8) is then given by

$$
\begin{aligned}
F(y) & =\frac{1}{16}\left[y^{2}(y+4)^{2}-b^{2}(b-4)^{2}\right], & G(x) & =1-\frac{1}{16} x^{2}(x+4)^{2}, \\
\kappa & =1, \quad \frac{1}{\mathcal{A}^{2} \ell^{2}}=1-\frac{1}{16} b^{2}(b-4)^{2}, & \mu & =\sqrt{q_{e}^{2}+q_{m}^{2}}=\frac{1}{4}, \quad 0 \leq b \leq 2 .
\end{aligned}
$$

Positivity of $1 / \mathcal{A}^{2} \ell^{2}$ restricts $b$ to range in $]-2(\sqrt{2}-1), 2(\sqrt{2}+1)[$, the boundary points and $b=2$ corresponding to the flat spacetime limit. Besides, $b \mapsto 4-b$ and $b \mapsto 2-\sqrt{4+4 b-b^{2}}$ leave the solution invariant, hence the range $0 \leq b \leq 2$ describes the fundamental domain of the solution and covers all the cases. We will therefore focus on that interval in the rest of the discussion. For $0<b<2, F(y)$ has the four zeros, $y_{0} \leq b-4<-b \leq y_{3}$. In the limiting case $b=0, F(y)$ has only two degenerate zeros in $y=-4$ and $y=0$. It corresponds to having the critical case $\mathcal{A}=1 / \ell$ where the cosmological attraction is balanced by the acceleration $\mathcal{A}$. In the other limiting case, $b=2$, the horizons at $y=b-4$ and at $y=-b$ coalesce and $F(y)$ has three zeros. It corresponds to the flat case limit where $\ell \rightarrow \infty$ and $F(y)=-G(y)$ (see figure 2.b).

A property of the $F$ and $G$ polynomials is that, as shown in appendix B, they become even under reflections through the $x=x_{0}$ and $y=x_{0}$ axes, respectively, once the thermal equilibrium condition is satisfied. Geometrically, this translates into an isometry that maps the $\left\{x-y<0 ; x<x_{0}\right\}$ region to the $\left\{x-y>0 ; x>x_{0}\right\}$ region, and the $\left\{x-y<0 ; x>x_{0}\right\}$ region to the $\left\{x-y>0 ; x<x_{0}\right\}$ region (see appendix $\mathrm{B}$ ). Hence, we can restrict the study of this spacetime to half of the coordinate space, for example $x>x_{0}$. For this reason, henceforth, we will consider the lukewarm solution only in the range $x_{0} \leq x \leq x_{B}$ and $y_{1} \leq y \leq y_{2}$ of the coordinates, corresponding to two static outer regions with AdS asymptotics as we shall shortly see (figure 4 ).

The horizons at $y=b-4$ and $y=-b$ have the same temperature $T_{H}=1 / \beta$ with

$$
\beta=\frac{4 \pi \sqrt{4+4 b-b^{2}}}{(4-b) b} .
$$

Moreover, the solution (2.9) has no conical singularities at $x=x_{B}$ (nor at $x=x_{A}$ ) if we take the period of $\phi$ to be

$$
\Delta \phi=\sqrt{2} \pi .
$$

Note that with this choice the solution is regular everywhere. Contrary to the standard claim, we thus have an explicit example of a charged AdS C-metric that is well behaved everywhere with horizons clothing the curvature singularity and no conical singularities. In the neutral case this observation was first pointed out in $[5,6]$.

\subsection{Black droplets and funnels in thermal equilibrium}

To show that the solutions studied in the previous subsection describe lukewarm black droplets and black funnels, we need to discuss first some properties of the system.

The range of the coordinates $x$ and $y$ is displayed in figure 4. As discussed in the previous paragraph, quadrants $I$ and $I V$ are isometric, and so are $I I$ and $I I I$. As we 
shall see, the points at $x=x_{0}$ are at infinite proper distance from any other point in the manifold, and we can therefore restrict the analysis to quadrants $I I$ and $I V$ without loss of generality. Henceforth we will restrict to the range $x_{0} \leq x \leq x_{B}$, the upper bound due to the fact that we must demand $G(x) \geq 0$ to have solutions with the right Lorentzian signature. The range $x>x_{B}$ represents the interior of the boundary black hole.

It is useful to rewrite the lukewarm solutions in Fefferman-Graham coordinates $(r, \chi)$, both to highlight the structure of their boundary and to study them in the context of AdS/CFT. This is a difficult task, but for our purpose it will be good enough to define $(r, \chi)$ close to the boundary; for the current solutions we find that they are obtained by the coordinate transformation

$$
y=x+\varepsilon \frac{1}{r}, \quad x=\chi-\varepsilon \frac{\mathcal{A}^{2} \ell^{2} G(\chi)}{r}, \quad \text { with }\left\{\begin{array}{lll}
\varepsilon=-1 & \text { if } & x-y>0, \\
\varepsilon=+1 & \text { if } & x-y<0 .
\end{array}\right.
$$

Here, $r$ is the holographic radial coordinate. Indeed, there is a curvature singularity at $r=0$ (i.e. $y= \pm \infty$ ) and the asymptotic AdS boundary is at $r \rightarrow \infty$ (i.e. $x=y$ ). The coordinate transformation in the polar coordinate, $x(\chi, r)$ was chosen to guarantee that the cross term between the radial and polar component of the metric vanishes at infinity up to order $1 / r^{2}$, i.e. $g_{r i} \sim \mathcal{O}\left(1 / r^{2}\right)$ as $r \rightarrow \infty$ (with $i$ being a spacelike boundary coordinate). These correspond to a perturbative version of the familiar Fefferman-Graham coordinates. As will be discussed later, this choice guarantees that the standard counterterm prescription for AdS spacetimes yields a finite action for the solutions.

The geometry can be foliated with surfaces of constant $r$, that we call $\mathcal{B}_{R}$. These are defined by $f(r)=r-R=0$ and have unit normal $n_{\mu}=\partial_{\mu} f /|\partial f|$. The induced metric on these surfaces, $\gamma_{\mu \nu}$, and their extrinsic curvature $K_{\mu \nu}$ are then

$$
\gamma_{\mu \nu}=g_{\mu \nu}-n_{\mu} n_{\nu}, \quad K_{\mu \nu}=\gamma_{\mu}^{\rho} \gamma_{\nu}^{\sigma} \nabla_{\rho} n_{\sigma}
$$

The boundary $\mathcal{B}$ of the lukewarm geometries is obtained by taking the hypersurface $\mathcal{B}_{R}$ to infinity, i.e. $R \rightarrow \infty$. The boundary metric $h_{a b}$, where Roman indices run over the boundary coordinates, is then defined by taking the inverse of $\gamma^{a b}$, which in our case is simply $\gamma_{a b}$, and rescaling it by the inverse power of its conformal factor, i.e.

$$
h_{a b}=\lim _{r \rightarrow \infty} \frac{\mathcal{A}^{2}}{r^{2}} \gamma_{a b} \Rightarrow d s_{\mathrm{bdy}}^{2}=-\mathcal{A}^{2} \ell^{2} F(\chi) d t^{2}+\frac{d \chi^{2}}{\mathcal{A}^{2} \ell^{2} F(\chi) G(\chi)}+G(\chi) d \phi^{2} .
$$

Close to the boundary $\chi$ tends to $x$, hence its range is $x_{0} \leq \chi \leq x_{B}$ (we are in quadrants $I I$ and $I V$ ). This coordinate plays the role of radial coordinate of the boundary metric. The resulting boundary geometry has some intriguing properties. First notice that it is not a solution of $d=3$ Einstein-Maxwell theory which indicates that there is an effective stress tensor, $8 \pi \mathcal{G}_{3} T_{a b}^{\mathrm{eff}}=\mathcal{R}_{a b}-\frac{1}{2} \mathcal{R} h_{a b}$ (with $\mathcal{R}_{a b}$ and $\mathcal{R}$ being the Ricci tensor and scalar associated with $h_{a b}$ ) living on the boundary $\mathcal{B}$. This is no surprise, since there is no dynamical gravity on the boundary: the conformal metric $h_{a b}$ describing the background on which the dual CFT fields propagate simply fixes (part of) the boundary conditions. Its geometry describes a boundary black hole with horizons at the zeros of $F(\chi)$. The 
inner horizon is at $\chi=y_{3}$ and the outer horizon is at $\chi=-b$. An investigation of the large radial behaviour of (2.14) confirms that it indeed describes a black hole with a well defined asymptotic region. This exercise will also allow us to finally understand why we choose only the lukewarm solutions that have a degenerate root of $G(x)$ at $x=x_{0}[5]$. Start by noticing that the proper distance between any two points at radial coordinates $\chi_{1}$ and $\chi_{2}=x_{0}$ is given by

$$
\Delta \chi=\frac{1}{\mathcal{A} \ell} \int_{\chi_{1}}^{x_{0}} \frac{d \chi^{\prime}}{\sqrt{F\left(\chi^{\prime}\right) G\left(\chi^{\prime}\right)}} .
$$

From the above expression it is clear that $\Delta \chi$ diverges if and only if $x=x_{0}$ is a double root of $G(x)$. This point is then at an infinite proper spatial distance from any other point with $\chi \neq x_{0}$ and we naturally identify $\chi=x_{0}$ as the asymptotic region of the boundary geometry. Moreover, in its neighborhood, the norm of the stationary Killing vector satisfies $\left\|\partial_{t}\right\| \rightarrow-1$, consistent with the proposed interpretation. In turn, the spatial part of (2.14) reduces to

$$
d s_{2}^{2}=\frac{d \chi^{2}}{\left(\chi-x_{0}\right)^{2}}+\left(\chi-x_{0}\right)^{2} d \phi^{2} .
$$

This is the metric on a Euclidean hyperboloid $\mathbb{H}^{2}$. Therefore, the boundary black hole asymptotes to $\mathbb{R} \times \mathbb{H}^{2}$.

It is useful to look into the bulk charged AdS C-metric in the neighborhood of the region $\chi \sim x_{0}$. We can zoom the bulk metric on this asymptotic region by taking an appropriate scaling limit. Concretely, take (2.1) with (2.9) and perform the scaling transformation [5]

$$
\epsilon \rightarrow 0, \quad \text { with } \quad t, y, X=\frac{x-x_{0}}{\epsilon} \text { and } \Phi=\frac{1}{2} \epsilon \phi \quad \text { fixed }
$$

which yields $\left(\mathcal{A}^{2} \ell^{2}\right.$ is given by $\left.(2.9)\right)$

$$
\begin{aligned}
A & =q_{e} \ell y d t+\frac{\sqrt{1-16 q_{e}^{2}}}{2 \mathcal{A}} X d \Phi, \\
d s^{2} & =\frac{1}{\mathcal{A}^{2}\left(x_{0}-y\right)^{2}}\left(-\mathcal{A}^{2} \ell^{2} F(y) d t^{2}+\frac{d y^{2}}{F(y)}+\frac{2}{X^{2}} d X^{2}+2 X^{2} d \Phi^{2}\right) .
\end{aligned}
$$

Take now the coordinate transformation,

$$
t=\frac{\sqrt{2}}{\ell} T, \quad y=x_{0}-\frac{\sqrt{2}}{\mathcal{A} \rho},
$$

to get

$$
\begin{aligned}
A & =\left(\sqrt{2} x_{0}-\frac{2}{\mathcal{A} \rho}\right) q_{e} d T+\frac{\sqrt{1-16 q_{e}^{2}}}{2 \mathcal{A}} X d \Phi, \\
d s^{2} & =-f(\rho) d T^{2}+\frac{d \rho^{2}}{f(\rho)}+\rho^{2}\left(\frac{d X^{2}}{X^{2}}+X^{2} d \Phi^{2}\right), \quad f(\rho)=\frac{\rho^{2}}{\ell^{2}}-1+\frac{(2 \mathcal{A})^{-2}}{\rho^{2}}
\end{aligned}
$$

with $\mathcal{A}$ given again by (2.9). Notice that the purely magnetic solution is obtained when one sets $q_{e}=0$ and one then has $q_{m}=1 / 4$, while the purely electric solution corresponds to set $q_{e}=1 / 4$ and thus $q_{m}=0$. This is a solution of AdS-Einstein-Maxwell theory [24]. 

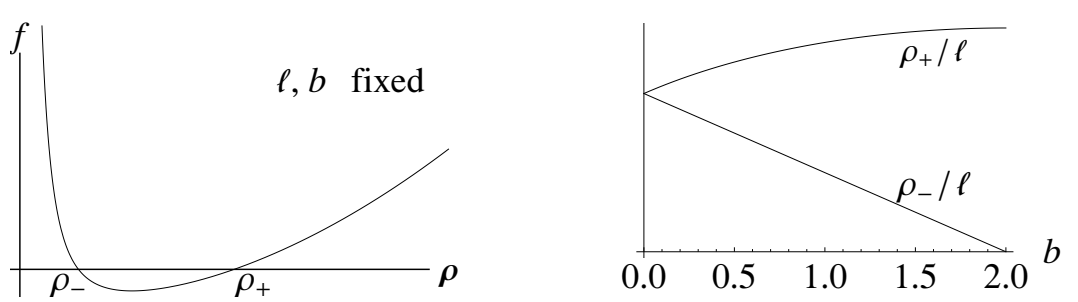

Figure 3. In the neighbourhood of $x=x_{0}$ the bulk lukewarm AdS C-metric is described by (2.20). It represents a charged hyperbolic black hole. The function $f(\rho)$ is represented in the left plot, and has two hyperbolic horizons at $\rho_{-}$and $\rho_{+}$. Their location as a function of the parameter $b$ is represented in right plot. For $b=0$, we have an extreme solution.

It describes a charged black hole with hyperbolic horizons, i.e. with $\kappa=-1$. Recall that the most general family of such a black hole is given by $f(\rho)=\frac{\rho^{2}}{\ell^{2}}-1+\frac{M}{\rho}+\frac{Q^{2}}{\rho^{2}}$. So for our solution $(2.20)$ one has $M=0$ and $Q=(2 \mathcal{A})^{-1}$. Although the mass parameter vanishes in this case, the geometry still describes a regular black hole with inner $\rho_{-}$and outer $\rho_{+}$ horizons clothing the curvature singularity, because it is now in the $\kappa=-1$ class. These horizons are located at $\rho_{ \pm}=\sqrt{4 \ell^{2}(1 \pm b) \mp \ell^{2} b^{2}} /(2 \sqrt{2})$ (see figure 3 ).

At this point we can finally discuss in detail the announced interpretation for our special family of lukewarm C-metrics. They can be interpreted as black droplets and/or black funnels in thermal equilibrium described by (2.1), (2.3) and (2.9). We can rewrite these solutions in terms of the Fefferman-Graham coordinates $(r, \chi)$ defined in (2.12). When the radial coordinate $r$ goes to infinity, one reaches the holographic boundary of the droplets and funnels. At this boundary the droplets and funnels induce a boundary black hole described by (2.14). The coordinate $\chi$ is a polar angle in the bulk, but in the boundary it plays the role of a radial coordinate. The asymptotic region of the boundary black hole is at $\chi=x_{0}$, and is at an infinite spatial proper distance from any other point in the bulk by (2.15). The black droplets and funnels live in range $x_{0} \leq x \leq x_{B}$. What remains are two quadrants $I I$ and $I V$, each of which fully describes a well-behaved solution (figure 4). The $\partial_{\phi}$ axis of rotation at $x=x_{B}$ for the solutions in quadrants $I I$ and $I V$ is free of conical singularities if we choose the period of $\phi$ to be (2.11). The axis $x=x_{0}$ is also regular and describes points that are at arbitrarily large distances from any other $\chi$. Quadrant $I I$ is defined by $y-x=1 / r>0$ while quadrant $I V$ has $x-y=1 / r>0$. The asymptotic holographic boundary is shared by these two quadrants, and is at $x-y=0$.

The solution of quadrant $I I$ describes a black funnel. Indeed, starting at point between $E$ and $A$ on the holographic boundary, we are in the exterior of the boundary black hole with an outer horizon represented by point $E$. As we enter into the bulk (towards smaller values of $\left.r=(y-x)^{-1}\right)$, we will first cross the outer horizon, $y=-b$, and then the inner horizon, $y=y_{3}$, of the black funnel, until we hit the curvature singularity at $y=+\infty(r=0)$. If we start at the holographic boundary already inside the inner horizon of the boundary black hole, i.e. at a point between $M$ and $N$, then as we move into the bulk we will remain inside the bulk black funnel until we reach the curvature singularity at $y=+\infty$. Therefore, this solution in quadrant $I I$ indeed describes a black funnel as sketched in figure 1.a. In that figure, we just represent the outer horizon of the black funnel. In the bulk and for 'large' 


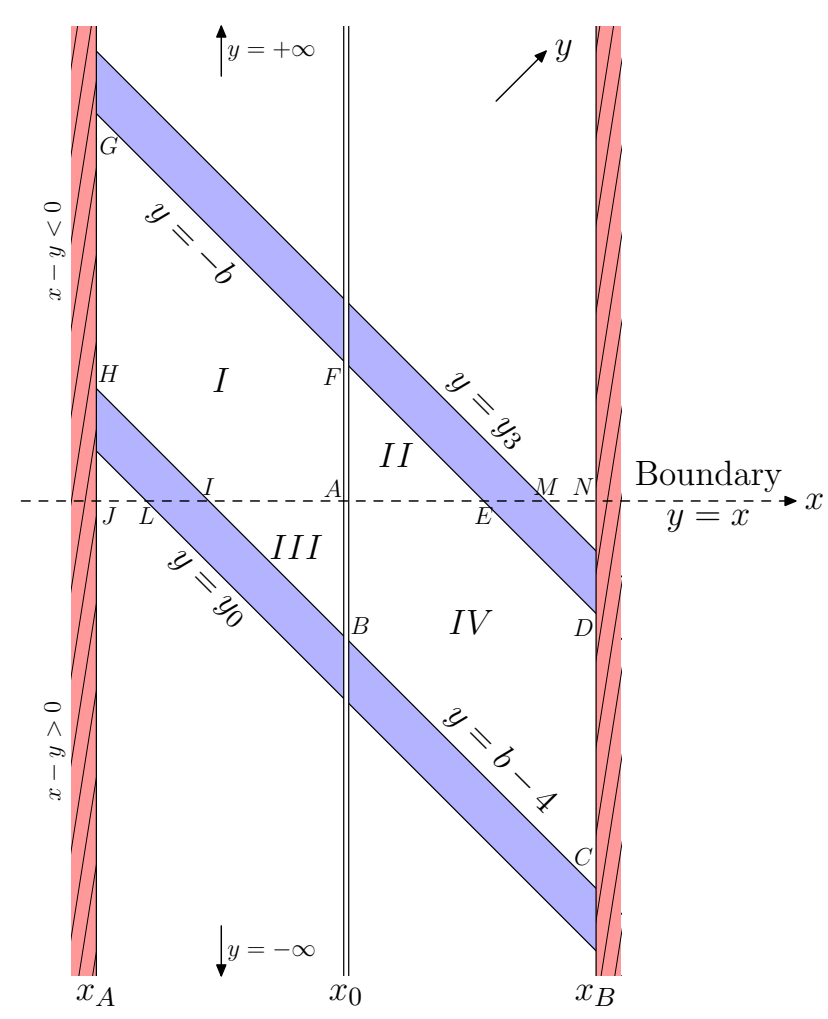

Figure 4. Black droplets (quadrants $I$ and $I V$ ) and black funnels (quadrants $I I$ and $I I I$ ) described by the lukewarm charged AdS C-metric (2.1), (2.3) and (2.9). The black funnel of quadrant $I I$ and the black droplet of quadrant $I V$ share the same boundary at $y=x$ and their outer horizons are at the same temperature. They are related through a symmetry transformation the solutions of quadrants $I I I$ and $I$. The holographic boundary point $A$ is at an infinite proper distance from any other point with $\chi \neq x_{0}$ and thus describes the asymptotic region of the boundary. The white regions are static, while the colored diagonal strips are between the inner and outer horizons.

values of $\chi$ in the neighborhood of $\chi \sim x_{0}$ (the 'shoulder region'), the funnel approaches the geometry of a hyperbolic black hole described by (2.20). At the holographic boundary, the horizons of the black funnel match the horizons of the boundary black hole.

On the other hand, the solution of quadrant $I V$ describes a black droplet, hovering over a planar black brane. Let us start at a point between $A$ and $E$ at the holographic boundary, i.e. between the asymptotic region (point $A$ ) and outside the outer horizon (point $E$ ) of the boundary black hole. We can dive into the bulk keeping away from the $D E$ horizon until we first cross the outer horizon $y=b-4$, then the inner horizon $y=y_{0}$, and finally hit the curvature singularity $y=-\infty(r=0)$ of the deformed hyperbolic black hole. Again, for 'large' values of $\chi$ in the neighborhood of $\chi \sim x_{0}$, this deformed hyperbolic black hole approaches the geometry of a hyperbolic black hole described by (2.20). Instead, we can start inside the inner horizon of the boundary black hole, i.e. in between points $M$ and $N$. As we enter into the bulk, we will at first stay inside the inner horizon $\left(y=y_{3}\right)$ of the black droplet. As we keep going deeper into the bulk, we will cross the outer horizon $(y=-b)$ of the black droplet. These horizons of the black droplet coincide with the horizons of 
the boundary black hole at the holographic boundary. At this stage we are outside any black hole horizon but, as we keep moving deeper into the bulk, we will cross the outer horizon $y=b-4$, then the inner horizon $y=y_{0}$, and finally we will hit the curvature singularity $y=-\infty$ of the deformed hyperbolic black hole. This description of the solution in quadrant $I V$ indeed corresponds to the black droplet interpretation that is sketched in figure 1.b. In this figure, we again just represent the outer horizon of the black holes of the system. It is important to notice that the black droplet of quadrant $I V$ and the black funnel of quadrant $I I$ share the same boundary, and that their outer horizons have the same temperature.

To finish this section we address the issue of the acceleration parameter source. In the standard interpretation of the C-metric as a pair of accelerated black holes [11], one always works in a range of coordinates where we choose the period of $\phi$ to eliminate a conical singularity at one of the poles. This leaves a singularity on the other pole of $\partial_{\phi}$ that is interpreted as a cosmic string or strut that sources the acceleration $\mathcal{A}$ of the black holes, as explained in the previous subsection. However, if we work instead in the subsector of C-metric solutions where $G(x)$ has a double root at $x=x_{0}$, this point is infinitely far apart from any other point $x$ in $x_{0} \leq x \leq x_{B}$. We can then choose the period of $\phi$ that eliminates the conical singularity at $x=x_{B}$; e.g. for the lukewarm solutions this period is given by (2.11). The potential deficit angle (2.6) at $x=x_{0}$ vanishes because $G^{\prime}\left(x_{0}\right)=0$ when $x_{0}$ is a double root: $x=x_{0}$ is at asymptotic infinity and the solution is then also regular here without any conical singularity/cosmic string. The fact that these solutions with a double root of $G(x)$ are regular everywhere is satisfying but the lack of a physical source that explains the origin of the acceleration parameter is puzzling. Futhermore, it is not clear how we can measure the acceleration parameter that characterizes these solutions. Indeed, although the mass and the charges of the solution are conserved charges that can be measured at infinity, we seem to lack an explicit measure of $\mathcal{A}$. Notice that this issue applies equally well to the uncharged solutions studied in $[5,6]$. It is important to try to understand this issue.

We think that the appropriate explanation borrows some ideas learned with a completely different solution, namely the dipole black rings [35]. In short, and in the simplest case, these are black ring solutions of supergravity that are electrically coupled to a twoform potential (or to a dual magnetic one-form in five dimensions) i.e. they create a field analogous to a dipole. The keypoint for our purposes is that these solutions are characterized by more parameters than conserved charges (which can be measured at infinity). In the simplest case, the five-dimensional dipole black ring is characterized by three parameters, but only has two conserved charges (mass and angular momentum). The third parameter is a non-conserved dipole charge that cannot be measured at infinity. Instead, to compute it we have to do a local operation where we consider an $S^{2}$ that encloses a section of the ring (that is locally a string). The dipole charge is then given by the electric field flux across this $S^{2}$.

The droplet/funnel system seems to have some resemblance with the dipole rings in the sense that it is also a solution that is characterized by more parameters than conserved charges. The acceleration parameter $\mathcal{A}$ is not a conserved charge. Instead, to determine 
it we also need to do a kind of a local measurement as follows. We already saw that in the neighborhood of $\chi \sim x_{0}$ the black droplets and black funnels share the same geometry, namely (2.20). In this region the geometry describes a hyperbolic black hole characterized only by its charge that is proportional to the acceleration, $Q=(2 \mathcal{A})^{-1}$. To measure $\mathcal{A}$, we can then make a local measurement of the decay of its associated electromagnetic field in the asymptotic region $r \rightarrow \infty$ and $\chi \rightarrow x_{0}$.

\section{Free energy of the black droplets and funnels}

The black funnel of quadrant $I I$ and the black droplet of quadrant $I V$ share the same holographic boundary, where they induce the same boundary metric. Furthermore, they are in thermal equilibrium since their outer horizons have the same temperature. Therefore, given the boundary data, both geometries contribute to the gravitational partition funtion. In this section, we shall compute the Euclidean action of the black funnel and the black droplet, thus showing which of the configurations dominates the thermal ensemble. We shall work only with the canonical ensemble for the magnetic solutions, for the reasons we will now detail.

Let us address the issue of regularity of the electromagnetic field for the black droplet. The charge and the associated chemical potential are relevant for the thermodynamic description of the system. Let us imagine that we had rotation instead of charge in our solutions. If the angular velocities of the two event horizons did not match, the Euclideanised solution would possess a conical singularity, as it would if the temperatures did not match. The charged case is different though, since the associated irregularities are not in the geometry itself. Nevertheless, the chemical potential in our black droplet solutions cannot be made to match between the black droplet and the deformed brane horizons. Since the two horizons cannot be in equibrium in this sense, we shall not work with the grand-canonical ensemble.

Consider the electromagnetic potential $A(2.3)$. Let us assume first that $q_{e}=0$, i.e. we have a magnetic solution. In the well known case of a spherical magnetic monopole, $A$ is required to be regular (e.g. $A^{2}$ finite) on each segment of the axis $\theta=0, \pi$. One can consider two patches, each covering one segment, on which a gauge is chosen such that $A$ vanishes on the axis. Then the consistency condition for gluing the two patches is that particle charges on the monopole background are quantised. In our case, we have a single segment of the rotation axis, $x=x_{B}$, but we also have the spatial limit $x=x_{0}$ on which $A^{2}$ diverges, unless a convenient gauge is chosen. Therefore, we also need two patches,

$$
x=x_{B}: \quad A \rightarrow A-d\left(\frac{q_{m}}{\mathcal{A}} x_{B} \phi\right), \quad x=x_{0}: \quad A \rightarrow A-d\left(\frac{q_{m}}{\mathcal{A}} x_{0} \phi\right),
$$

whose gluing leads to a quantisation condition on particle charges $e$,

$$
e \frac{q_{m}}{\mathcal{A}}\left(x_{B}-x_{0}\right)=2 \pi n
$$

where $n$ is an integer. 
Consider now the electrically charged solutions. As we mentioned, there is a difference in the electric potential $\phi_{E}=-q_{e} \ell y$ in (2.3) between the two horizons, located at $y=-b$ and $y=b-4$. The corresponding electric field would lead to a current between the horizons if any charged particles were present. (There is always at least one type of charged particle in Einstein-Maxwell theory, which is a mini black hole produced as a quantum fluctuation, but this effect is suppressed as $1 / N$ in the AdS/CFT correspondence.) Notice that the usual quantisation procedure, as discussed above for the magnetic case, fails here. One could consider two patches, such that each covers one horizon, and on each patch there is a simple gauge choice which makes $A_{\mu}$ regular at that horizon. However, that gauge transformation would depend on the time coordinate. Since, in the Euclideanised spacetime, we have $t \rightarrow-i \tau$, we cannot make sense of the corresponding gauge transformations for charged particles in the system. Since we cannot find a meaningful way of working with the electric solutions, even in the canonical ensemble, we shall consider magnetic solutions only, i.e. $q_{e}=0$.

We now proceed to the computation of the free energy of the magnetic black funnel and black droplet. We will be comparing these solutions at the same values of the temperature and of the magnetic charge, i.e. we consider here the canonical ensemble. The relevant thermodynamic potential is therefore the Helmholtz free energy.

The Helmholtz free energy $\mathcal{F}$ of a solution is given by the ratio of the Euclidean action of the solution $I$ and its thermal period $\beta, \mathcal{F}=I / \beta$. The Euclidean action includes the AdS-Einstein-Maxwell integral over the bulk $\mathcal{M}, I_{\text {bulk }}\left[g_{\mu \nu}, A_{\mu}\right]$, plus the York-GibbonsHawking surface integral over the boundary $\partial \mathcal{M}, I_{\mathrm{YGH}}\left[g_{\mu \nu}\right]$. The latter is required so that upon variation with metric fixed at the boundary, the action yields Einstein's equations. This is not the whole story. Since we are in an asymptotically AdS background, the sum of these contributions, $I_{\mathrm{bulk}}+I_{\mathrm{YGH}}$, diverges because the volumes of both $\mathcal{M}$ and $\partial \mathcal{M}$ are infinite (and the integrands are nonzero). The correct and finite computation of the gravitational action then requires regulating the action. In the counterterm subtraction approach, the divergences are eliminated by adding a counterterm contribution to the action, $I_{\mathrm{ct}}\left[\gamma_{a b}\right][36-39]$. This is an extra surface integral that depends only on the induced boundary metric $\gamma_{a b}$ and its derivatives. If we work in Fefferman-Graham coordinates, the expression for $I_{\mathrm{ct}}\left[\gamma_{a b}\right]$ is universal, depending only on $\ell$ and the spacetime dimension.

The finite Euclidean gravitational action for a solution of AdS-Einstein-Maxwell theory, and including only the counterterms that make a contribution in four dimensions, is then given by [36-39]

$$
I=I_{\mathrm{bulk}}\left[g_{\mu \nu}, A_{\mu}\right]+I_{\mathrm{YGH}}\left[g_{\mu \nu}\right]+I_{\mathrm{ct}}\left[\gamma_{a b}\right]
$$

with

$$
\begin{aligned}
I_{\mathrm{bulk}} & =-\frac{1}{16 \pi \mathcal{G}} \int_{\mathcal{M}} d^{4} x \sqrt{g}\left(R+\frac{6}{\ell^{2}}-F_{\alpha \beta} F^{\alpha \beta}\right), \quad I_{\mathrm{YGH}}=-\frac{1}{8 \pi \mathcal{G}} \int_{\partial \mathcal{M}} d^{3} x \sqrt{\gamma} K, \\
I_{\mathrm{ct}} & =\frac{1}{8 \pi \mathcal{G}} \int_{\partial \mathcal{M}} d^{3} x \sqrt{\gamma}\left(\frac{2}{\ell}+\frac{\ell}{2} \mathcal{R}\right) .
\end{aligned}
$$

Here, $R$ is the Ricci scalar of the bulk Euclidean metric $g_{\mu \nu}, \mathcal{R}$ is the Ricci scalar of the induced boundary metric $\gamma_{a b}$, and $K=\gamma^{a b} K_{a b}$ is the trace of the extrinsic curvature of 
the boundary $\partial \mathcal{M}$ as embedded in $\mathcal{M}$ defined in (2.13). ${ }^{2}$

A word of caution is in order here. It is widely assumed in the literature that the expression for $I_{\mathrm{ct}}$ as written in (3.4) provides a finite covariant definition of the gravitational action for asymptotically AdS spaces. That is, it is often assumed that one may use this expression for $I_{\mathrm{ct}}$ to regulate the action for any choice of coordinates on any asymptotically AdS solution. However, this is not quite correct [41]. Indeed the standard counterterms as written in (3.4) are derived using a Fefferman-Graham coordinate system. These counterterms, defined on hypersurfaces of constant Fefferman-Graham (FG) radial coordinate, are not necessarily the correct counterterms on the hypersurfaces of constant radial coordinate in all other coordinate systems. If we choose instead to work in a coordinate system where the radial coordinate does not agree with the radial FG coordinate we should redo the asymptotic analysis for the new radial coordinate from the very beginning using a regulator for this new radial coordinate and rederive the appropriate counterterms.

This is an important issue in the current analysis because we find that if we work with the $\mathrm{C}$-metric in the $\{t, r, x, \phi\}$ coordinate system, i.e. with $y=x \pm 1 / r$ and $x=\chi$ instead of (2.12), then the total action with the counterterm as written in (3.4) does not yield a finite result. This is a particular case where the expression (3.4) derived using a FG coordinate system does not hold on another coordinate system. Another example where a similar situation occurs is the Kerr-Newman black hole: if we work in the standard Boyer-Lindquist coordinate system, expression (3.4) for the counterterm action also fails to render the total action finite [41].

We choose to work with the well established FG counterterm action (3.4). The appropriate perturbative definition of the FG coordinate system is that, up to sufficient order in $1 / r$, the coefficient of the radial line element should not depend on the polar angle, and there cannot be a mixed term between the FG radial $r$ and angular the $\chi$ coordinate. The criteria to decide which coefficients of this series expansion in $1 / r$ vanish is the requirement that the gravitational action (3.3) is finite. With this prescription, we find that the Fefferman-Graham coordinates for the C-metric are those written in (2.12). The coordinate transformation in the polar angle $x(\chi, r)$ was chosen to guarantee that the cross term between the radial and polar component of the metric vanishes at infinity up to order $1 / r^{2}$, i.e. $g_{r i} \sim \mathcal{O}\left(1 / r^{2}\right)$ as $r \rightarrow \infty$ (with $i$ being a spacelike boundary coordinate). This is enough to obtain a finite action as we confirm next.

To evaluate the bulk and surface integrals in (3.4) we need to define the position of the horizons, of the boundary and of the azimuthal axis in the Fefferman-Graham coordinates (2.12). The map between the black droplet/funnel regions in the $(x, y)$ coordinates and the $(\chi, r) \mathrm{FG}$ coordinates is represented in figure 5 .

\footnotetext{
${ }^{2}$ For a non-zero electric charge, the Hawking-Ross surface integral, $I_{\mathrm{HR}}\left[g_{\mu \nu}, A_{\mu}\right]$, also contributes to the total action in the canonical ensemble [40]. This term allows us to impose fixed electric charge $q_{e}$ as a boundary condition at infinity, and is required by the electromagnetic duality. Denoting with a superscript $(e)$ the electric sector of the electromagnetic field, the total Euclidean action is given by $I=$ $I_{\mathrm{bulk}}+I_{\mathrm{YGH}}+I_{\mathrm{HR}}+I_{\mathrm{ct}}$, with

$$
I_{\mathrm{HR}}=-\frac{1}{4 \pi \mathcal{G}} \int_{\partial \mathcal{M}} d^{3} x \sqrt{\gamma} F^{(e) \mu \nu} A_{\mu}^{(e)} n_{\nu}
$$



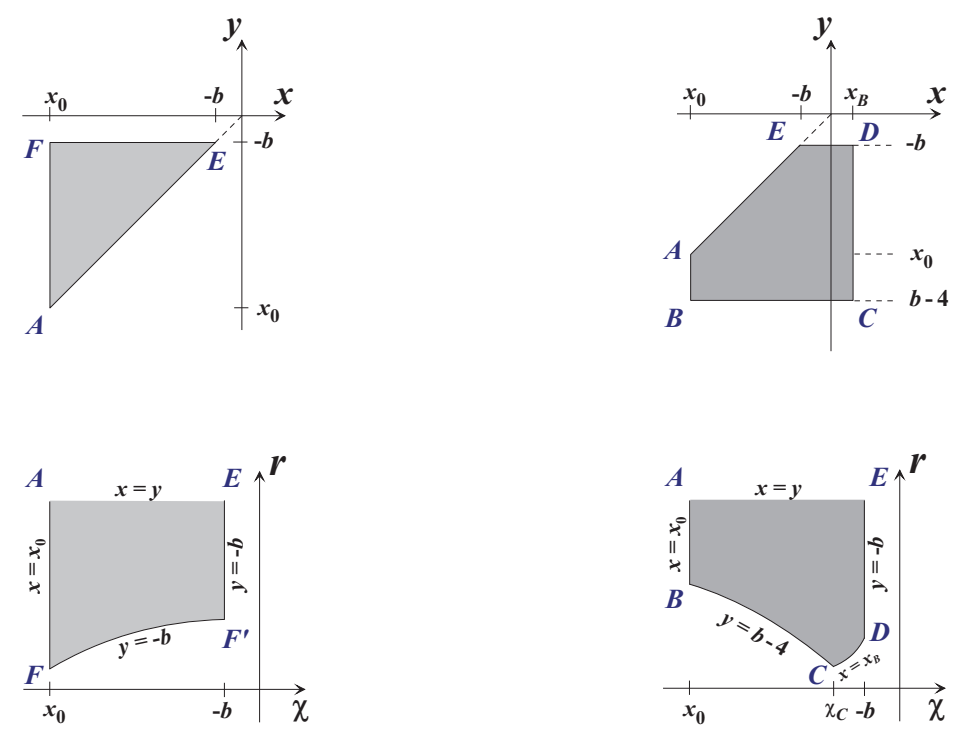

Figure 5. Map between the boundaries of the black funnel and black droplet in the original coordinates $(x, y)$ and the $(\chi, r)$ Fefferman-Graham coordinates defined in (2.12). The left panel describes the black funnel of quadrant $I I$, whose single horizon is split into two branches in the $(\chi, r)$ coordinates: $F F^{\prime}$ and $F^{\prime} E$. This solution shares its asymptotic holographic boundary $A E$ with the black droplet of quadrant $I V$ described in the right panel. The black droplet and black brane horizons correspond to the $D E$ and $B C$ curves, respectively, and are disjoint.

The left panel of figure 5 shows this map for the black funnel of quadrant $I I$. The closed curve $[A E F]$ is in correspondence with the associated curve in quadrant $I I$ of figure 4 . Particular care must be taken when mapping the segment $[E F]$, representing the horizon, to $(r, \chi)$ coordinates, since its intersection point with asymptotic infinity $F$ is resolved by the map (2.12) into a curve $\left[F F^{\prime}\right]$. To see this, we cut the spacetime at a coordinate distance $\varepsilon>0$ from the horizon, so that the triangle in the upper figure 5.a ends on a segment $[\hat{E}, \hat{F}]$ at $y=-b-\varepsilon$. This segment is mapped to a smooth curve in the FG coordinate plane, but as the regulator is removed in the limit $\epsilon \rightarrow 0$, this curve becomes non-differentiable at the point $F^{\prime}$. This breaks the full curve $[E F]$ into the curve $\left[F F^{\prime}\right]$ described by $r(\chi)=-\frac{\mathcal{A}^{2} \ell^{2} F(\chi)}{\chi+b}$, and the vertical line $\left[F^{\prime} E\right]$ at $\chi=-b$. Since the full curve describes the boundary of the integration region and it remains piecewise differentiable, the appearance of this singularity is harmless and the integration can be performed directly over the $\left[A F F^{\prime} E\right]$ region of figure 5.a in FG coordinates. This black funnel shares its asymptotic boundary $[A E]$ with the black droplet of quadrant $I V$. The right panel of figure 5 presents the map $(x, y) \rightarrow(\chi, r)$ for the latter black droplet. The closed curve $[A B C D E]$ is in correspondence with the associated curve in quadrant $I V$ of figure 4 . The curve $B C$ is described by $r(\chi)=\frac{\mathcal{A}^{2} \ell^{2} F(\chi)}{\chi-b+4}$. The curve $C D$ is described by $r(\chi)=\frac{\mathcal{A}^{2} \ell^{2} G(\chi)}{x_{B}-\chi}$. These two curves meet at point $C$, for which $\chi=\chi_{C}$.

With this information, we can finally compute the integrals in (3.4). The bulk integrals 

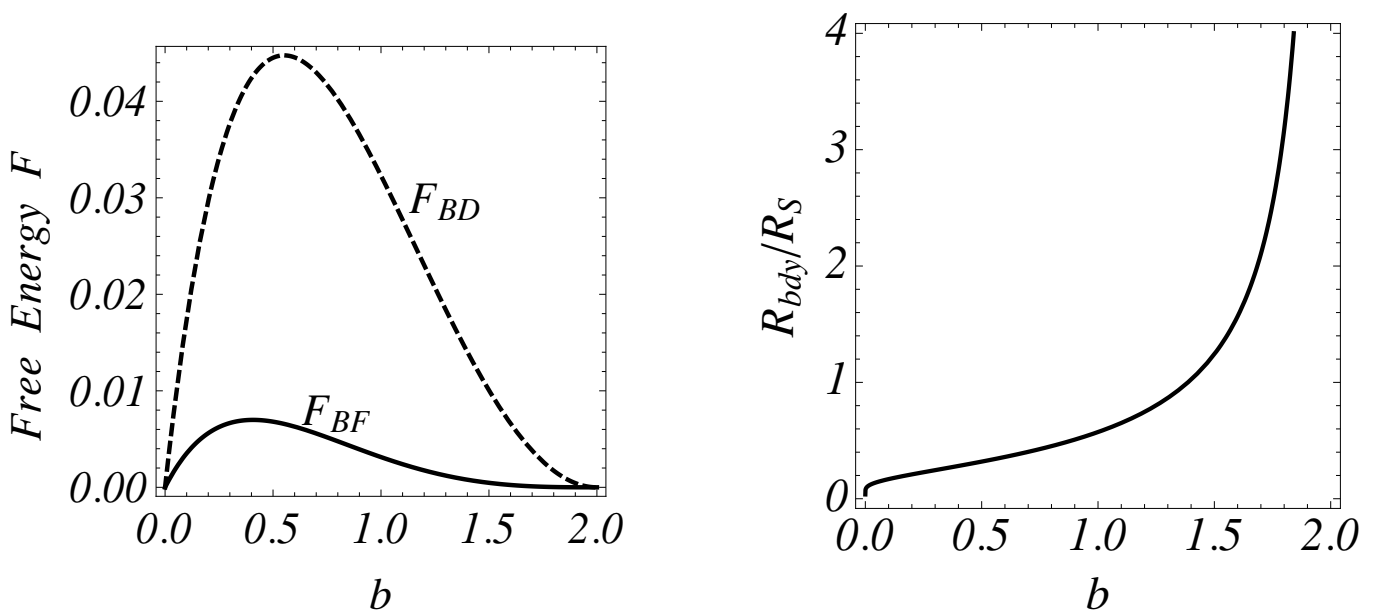

Figure 6. Left panel: Free energy for the black droplets $(B D)$ and black funnels $(B F)$ as a function of the parameter $b$ for fixed $\ell(\mathcal{G}=1)$. The free energies of both solutions vanish at $b=0$ because the temperature vanishes there, while the free energies are zero at $b=2$ because the associated Euclidean actions vanish there. Right panel: Size of the boundary black hole, $R_{\text {bdy }}$ vs. the distance $R_{\mathrm{S}}$ between the "shoulder" of the hyperbolic black brane or black funnel (see figure 1) and the holographic boundary.

$\int_{\mathcal{M}}$ in $I_{\text {bulk }}$ for the black funnel in quadrant $I I$ and the black droplet in quadrant $I V$ have the following schematic structure,

Black funnel in $I I: \quad \int_{\mathcal{M}} \rightarrow \int_{0}^{\beta} d \tau \int_{0}^{\Delta \phi} d \phi \int_{x_{0}}^{-b} d \chi \int_{-\frac{\mathcal{A}^{2} \ell^{2} F(\chi)}{\chi+b}}^{\infty} d r$

Black droplet in $I V: \quad \int_{\mathcal{M}} \rightarrow \int_{0}^{\beta} d \tau \int_{0}^{\Delta \phi} d \phi\left[\int_{x_{0}}^{\chi_{C}} d \chi \int_{-\frac{\mathcal{A}^{2} \ell^{2} F(\chi)}{b-4-\chi}}^{\infty} d r+\int_{\chi_{C}}^{-b} d \chi \int_{\frac{\mathcal{A}^{2} \ell^{2} G(\chi)}{x_{B}-\chi}}^{\infty} d r\right]$,

and the boundary integrals $\int_{\partial \mathcal{M}}$ in $I_{\mathrm{YGH}}$ and $I_{\mathrm{ct}}$ are given by the same integration structure but with the radial integration removed. In these integrals, the thermal period $\beta$ is given by (2.10) and the azimuthal period $\Delta \phi$ is given by (2.11).

Evaluating these integrals we finally find that the total, finite, action for the black droplets and black funnels is given by

$$
\begin{aligned}
\text { Black funnel in } I I: \quad I_{\mathrm{BF}} & =\frac{\pi(2-b)^{3}}{64 \sqrt{2} \mathcal{G}}, \\
\text { Black droplet in } I V: & I_{\mathrm{BD}}=\frac{\pi(2-b)^{2}(b-2+8 \sqrt{2})}{64 \sqrt{2} \mathcal{G}} .
\end{aligned}
$$

We can know compare the Helmholtz free energy $\mathcal{F}=I / \beta$ of the pairs of black droplets/funnels that share the same holographic boundary, charge and temperature. This is done in the left panel of figure 6. The black funnels always have a smaller free energy than the black droplets and therefore they always dominate the partition function. In particular, there is no phase transition between these two solutions, except possibly at $b=2$ 

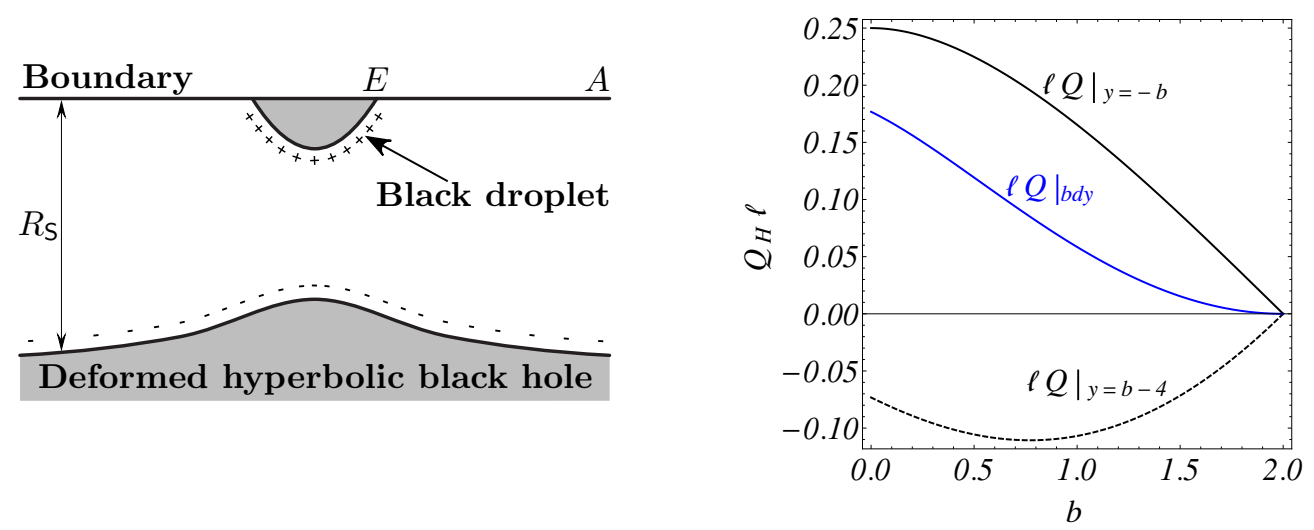

Figure 7. Left panel: The distance $R_{\mathrm{S}}$ between the "shoulder" region of the planar black hole and the boundary, and the distribution of charges on the horizons. Right panel: Charge of the black droplet horizon $\left.\ell Q\right|_{y=-b}$, charge of the deformed hyperbolic horizon $\left.\ell Q\right|_{y=b-4}$ and charge of the boundary black hole horizon $\left.\ell Q\right|_{\mathrm{bdy}}$. The latter satisfies (3.17) and also gives the black funnel horizon charge.

where the two free energies vanish. However, at this $b=2$ point the two horizons merge, leaving no static external spacetime region.

This conclusion is worth of some discussion. The notions of black funnels and black droplets and their interpretation were first introduced in [5]. Explicit examples of droplets and funnels, constructed out of the (uncharged) AdS C-metric, were also presented in [5, 6]. In these works it was advocated that there should be a phase transition between black funnels and droplets. For asymptotically flat boundary black holes, the transition point is expected to be determined by the ratio $R_{\mathrm{bdy}} / R_{\mathrm{S}}$. Here, $R_{\mathrm{bdy}}$ is the typical size of the boundary black hole, and $R_{\mathrm{S}}$ is the distance between the "shoulder" of the planar black brane or black funnel and the holographic boundary, as shown in the left panel of figure 7 (in the neutral case this distance is given by the inverse of the temperature $R_{\mathrm{S}} \sim 1 / T_{H}$ ). Ref. [5] argues that one should expect the black droplets to be dominant for $R_{\text {bdy }} / R_{\mathrm{S}} \ll 1$ and that the black funnels should dominate on the complementary regime, $R_{\mathrm{bdy}} / R_{\mathrm{S}} \gg 1$. However, the authors of $[5,6]$ did not test their prediction against the results for the free energy of their explicit droplets and funnels. The reason is that the black droplets constructed out of their uncharged AdS C-metric can never be in thermal equilibrium, i.e. the droplet and deformed hyperbolic horizon can never be at the same temperature when the charge vanishes. As we explicitly found, this is no longer the case when we switch on the Maxwell field: the magnetic lukewarm black droplets are in thermal equilibrium. This allowed us to compare the free energies for the magnetic lukewarm black droplets and funnels, and we found no sign of a phase transition. Our boundary black holes asymptote to $\mathbb{R} \times \mathbb{H}^{2}$ (and are charged) instead of being asymptotically flat and thus the phase transition estimative of $[5,6]$ is not expected to hold. It is nevertheless interesting to check what happens. We will do it trying to follow as close as possible the arguments of $[5,6]$.

Consider first the boundary black hole size $R_{\mathrm{bdy}}$. Since our boundary black holes asymptote to $\mathbb{R} \times \mathbb{H}^{2}$, circles of constant radial coordinate $\chi$ shrink as $\chi$ increases. Therefore, 
we should not take, e.g. the proper size of constant $\chi$ circles as a measure of $R_{\mathrm{bdy}}$. On the other hand, the volume outside the boundary horizon is finite and decreases as the black hole size increases. ${ }^{3}$ Therefore a good measure of $R_{\text {bdy }}$ seems to be the inverse of this volume, i.e.

$$
R_{\mathrm{bdy}} \simeq\left[\int_{0}^{\sqrt{2} \pi} d \phi \int_{x_{0}}^{-b} d \chi \sqrt{\sigma}\right]^{-1}
$$

where $\sigma$ is the determinant of the induced metric $\sigma_{a b}=h_{a b}+N_{a} N_{b}$ on a timelike surface $f(t)=t-t_{0}=0$, with normal $N_{a}=-\partial_{a} f /|\partial f|$. (See (2.14), (2.11), and recall that for quadrants $I I$ and $I V$ the outer horizon is at $\chi=-b$.) To find $R_{S}$, i.e. the distance between the "shoulder" of the hyperbolic black brane or black funnel (see figure 1) and the holographic boundary, recall that the asymptotic "shoulder" region is described by (2.20). Introducing the new holographic coordinate $z=-\ell^{2} / \rho$, the gravitational field of $(2.20)$ reads

$$
d s^{2}=\frac{\ell^{2}}{z^{2}}\left[-f(z) d T^{2}+\frac{d z^{2}}{f(z)}+\ell^{2}\left(\frac{d X^{2}}{X^{2}}+X^{2} d \Phi^{2}\right)\right] \quad \text { with } \quad f(z)=1-\frac{z^{2}}{\ell^{2}}+\frac{1}{4 \mathcal{A}^{2} \ell^{2}} \frac{z^{4}}{\ell^{4}} .
$$

The holographic asymptotic boundary is at $z=0$ and the (outer) horizon position, $z=$ $z_{+} \equiv R_{\mathrm{S}}$, determines the desired distance between the holographic boundary and the brane/funnel "shoulder": 4

$$
R_{\mathrm{S}}=\sqrt{2} \mathcal{A} \ell \sqrt{1-\sqrt{1-\frac{1}{\mathcal{A}^{2} \ell^{2}}}} .
$$

The ratio $R_{\mathrm{bdy}} / R_{\mathrm{S}}$ for the families of funnels and droplets that we analyzed is plotted in the right panel of figure 6 . So, if the arguments of $[5,6]$ were valid also for asymptotically hyperbolic boundary black holes, we would expect the black droplets to dominate a low values of $b\left(R_{\mathrm{bdy}} \rightarrow 0\right.$ as $\left.b \rightarrow 0\right)$. This is not the case, as the left panel of figure 6 shows. In sum, the outcome of our exercise emphasizes that the phase transition estimative of [5] does not hold for boundary black holes that asymptote to $\mathbb{R} \times \mathbb{H}^{2}$, as pointed out in [6].

To have a better physical understanding of our results, note that the charge of the system contributes to the fact that black funnels dominate the partition function. A good strategy to confirm this statement is to look at the charges of the black funnel and droplet horizons, as defined by the pullback of the Maxwell field strength on the horizon hypersurface. As before, we will consider the magnetic charge only, i.e. $q_{e}=0$. In these conditions, the 2-form associated with the Maxwell potential (2.3), written in terms of the Fefferman-Graham coordinates (2.12), is

$$
F=\frac{q_{m}}{\mathcal{A} r^{2}}\left[\varepsilon \mathcal{A}^{2} \ell^{2} G(\chi) d r \wedge d \phi+r\left(r-\varepsilon \mathcal{A}^{2} \ell^{2} G^{\prime}(\chi)\right) d \chi \wedge d \phi\right]
$$

where $\varepsilon=1$ in quadrant $I I, \varepsilon=-1$ in quadrant $I V$; see (2.12).

\footnotetext{
${ }^{3}$ We thank Don Marolf for this observation.

${ }^{4}$ One could refine the definition of $R_{\text {bdy }}$ by using the inverse temperature of the hyperbolic brane (3.9) or the proper distance between the planar black brane and the black droplet instead of the holographic distance $z_{+}$. However, this does not affect qualitatively our discussion.
} 
We can consider the pullback of $F$ to a hypersurface $r=R(\chi)$, which yields

$$
\left.F\right|_{r=R(\chi)}=\rho_{R} d \chi \wedge d \phi, \quad \text { where } \quad \rho_{R}=\frac{q_{m}}{\mathcal{A}}\left[1-\varepsilon \mathcal{A}^{2} \ell^{2}\left(\frac{G(\chi)}{R(\chi)}\right)^{\prime}\right]
$$

is the charge density of a $r=R(\chi)$ hypersurface. Similarly we consider the pullback of $F$ to a hypersurface of constant $\chi$,

$$
\left.F\right|_{\chi=\text { const }}=\rho_{\chi} d r \wedge d \phi, \quad \text { where } \quad \rho_{\chi}=\frac{\varepsilon q_{m} \mathcal{A} \ell^{2} G(\chi)}{r^{2}}
$$

is the charge density of a constant $\chi$ hypersurface.

We can now compute the charge of the several horizons. In view of (2.9), hereafter we set $q_{m}=1 / 4$. Start with the charge measured asymptotically outside the boundary black hole horizon, which is given by

$$
\mathcal{Q}_{\text {bdy }}=\left.\frac{1}{4 \pi} \int_{0}^{\sqrt{2} \pi} d \phi \int_{x_{0}}^{-b} d \chi \rho_{R}\right|_{R \rightarrow \infty}=\frac{\ell}{32 \sqrt{2}}(b-2)^{2} \sqrt{4+4 b-b^{2}} .
$$

This is the charge of the holographic fluid outside the horizon. We can check that it matches the charge of the black funnel horizon. For the black funnel of quadrant $I I$, the charge includes a contribution from the surface $F F^{\prime}$ plus a contribution from the surface $F^{\prime} E$ in the left panel of figure 5 , and yields

$$
\mathcal{Q}_{B F}=\frac{1}{4 \pi} \int_{0}^{\sqrt{2} \pi} d \phi\left[\left.\int_{x_{0}}^{-b} d \chi \rho_{R}\right|_{R=-\frac{\mathcal{A}^{2} \ell^{2} F(\chi)}{\chi+b}}+\left.\int_{\frac{4 b(4-b)}{(2-b)\left(4+4 b-b^{2}\right)}}^{\infty} d r \rho_{\chi}\right|_{\chi=-b}\right]=\mathcal{Q}_{\mathrm{bdy}} .
$$

(Notice that this horizon charge does not agree, as expected, with the charge parameter $q_{m}$ of the black funnel.)

Consider now the black droplet system of quadrant $I V$. It has two horizons (and associated charges): one is the horizon of the droplet and the other of the deformed hyperbolic black hole. The charge of the droplet (see $E D$ in the right panel of figure 5) and of the hyperbolic (see $B C$ in the same figure) horizons are, respectively,

$$
\begin{gathered}
\left.\mathcal{Q}\right|_{y=-b}=\left.\frac{1}{4 \pi} \int_{0}^{\sqrt{2} \pi} d \phi \int_{\frac{1}{2(\sqrt{2}-1)+b}}^{\infty} d r \rho_{\chi}\right|_{\chi=-b}=-\frac{\ell(4-2 \sqrt{2}+\sqrt{2} b)\left(8+4 b-6 b^{2}+b^{3}\right)}{64 \sqrt{4+4 b-b^{2}}}<0, \\
\left.\mathcal{Q}\right|_{y=b-4}=\left.\frac{1}{4 \pi} \int_{0}^{\sqrt{2} \pi} d \phi \int_{x_{0}}^{\chi C} d \chi \rho_{R}\right|_{R=-\frac{\mathcal{A}^{2} \ell^{2} F(\chi)}{b-4-\chi}}=\frac{\ell}{16}(2-b) \sqrt{4+4 b-b^{2}}>0 .
\end{gathered}
$$

So the droplet and the deformed hyperbolic horizons have charges of opposite sign such that their sum gives, as it should, the boundary charge (outside the boundary black hole horizon),

$$
\mathcal{Q}_{B D}=\left.\mathcal{Q}\right|_{y=-b}+\left.\mathcal{Q}\right|_{y=b-4}=\mathcal{Q}_{\text {bdy }}
$$

This is an important observation that sheds light on the interpretation of our results. Indeed, since the droplet and deformed hyperbolic horizons have charges of opposite sign, these two black holes have an extra electromagnetic attraction with respect to the case 
where the horizons are uncharged. Due to this extra attraction, we expect that the free energy is minimised by the existence of a single horizon, and so the charged black funnels should extend their regime of domination at the expense of the black droplets. For the solutions that we studied, we find that the black funnels actually dominate over the entire parameter range.

\section{Holographic interpretation of black droplets and funnels}

The stress tensor of the field theory that lives in the AdS boundary of the black droplets and funnels is given by the holographic stress tensor. This tensor is the starting point to discuss the field theory description of the black droplets and funnels in thermal equilibrium.

The induced stress tensor associated with the action (3.3) is given by the variation of the action with respect to the induced boundary metric $\gamma^{a b}, \mathcal{T}_{a b}=\frac{2}{\sqrt{-\gamma}} \frac{\delta I}{\delta \gamma^{a b}}$, yielding ${ }^{5}$

$$
-8 \pi \mathcal{G} \mathcal{T}_{a b}=K_{a b}-\gamma_{a b} K+\frac{2}{\ell} \gamma_{a b}-\ell\left(\mathcal{R}_{a b}-\frac{1}{2} \mathcal{R} \gamma_{a b}\right)
$$

Since the induced boundary metric $\gamma_{a b}$ diverges near the boundary, this induced stress tensor vanishes at the boundary. In the standard AdS/CFT dictionary, the finite expectation value for the holographic stress tensor is obtained by rescaling the induced stress tensor by a power of the metric conformal factor,

$$
\left\langle T_{a b}\right\rangle=\lim _{r \rightarrow \infty}-\frac{\varepsilon r}{\mathcal{A}} \mathcal{T}_{a b}
$$

where $\varepsilon=+1$ in sectors II and III, and $\varepsilon=-1$ in sectors III and IV. ${ }^{6}$ After using the property $F^{\prime \prime \prime}(\chi)=12 \mu+24\left(q_{e}^{2}+q_{m}^{2}\right) \chi$ of the polynomial $F$, we obtain

$$
\left\langle T^{a}{ }_{b}\right\rangle=\frac{\mathcal{A} \ell^{3}}{8 \pi \mathcal{G}}\left[\mu+2\left(q_{e}^{2}+q_{m}^{2}\right) \chi\right] \operatorname{diag}\{G(\chi)-2 F(\chi), F(\chi)+G(\chi), F(\chi)-2 G(\chi)\} .
$$

In the Euclidean metric there is a symmetry between $x$ and $y$ and this is reflected in the fact that the signs and factors of two flip between the $t t$ and $\phi \phi$ components of $\left\langle T^{a}{ }_{b}\right\rangle$ as we pass from the horizon to the axes. Note that due to the relation $F(\chi)+G(\chi)=(\mathcal{A} \ell)^{-2}$, the holographic stress tensor is traceless, $\left\langle T_{a}^{a}{ }_{a}\right\rangle=h^{a b}\left\langle T_{a b}\right\rangle=0$. This is a consequence of the fact that the boundary field theory is odd dimensional, and thus there is no conformal anomaly.

\footnotetext{
${ }^{5}$ This expression (4.1), and (4.2), for the holographic tensor is derived from the Euclidean action (3.3) with the Fefferman-Graham counterterms. This same expression also yields the correct final answer for the stress tensor if we work with the coordinates $\chi=x, y=x \pm 1 / r$, instead of (2.12), even though the action is then not finite. This is because the formula for the stress tensor is boundary covariant. A similar situation occurs with the Kerr-Newman black hole: if (4.1) is evaluated in the Boyer-Lindquist coordinate system, the correct stress tensor is obtained notwithstanding that the action with the counterterm contribution (3.4) diverges in this coordinate system [41].

${ }^{6}$ The $-\varepsilon$ factor guarantees that the black funnel has the same $\left\langle T_{a b}\right\rangle$ as the black droplet and that this reduces to the $\left\langle T_{a b}\right\rangle$ of a perfect fluid asymptotically. This overall minus sign in the conformal factor is irrelevant for the boundary metric (2.14) since one uses the square of the conformal factor to obtain it.
} 
The form of the stress tensor (4.3) is that of a thermal gas of massless radiation. In general (4.3) does not correspond to a perfect fluid, $\left\langle T_{a b}\right\rangle=P(\chi)\left(h_{a b}+3 u_{a} u_{b}\right)$. However, near the asymptotic region of the holographic boundary, $\chi \sim x_{0}, G(\chi)$ vanishes and (4.3) does reduce to the stress tensor of a perfect fluid. In this region, the boundary metric approaches $\mathbb{R} \times \mathbb{H}^{2}$, and the bulk solution is described by the geometry of the hyperbolic black hole (2.20). The dual field theory for such a black hole has been discussed in detail in [42]. There, it was shown that this black hole minimizes the free energy for all temperatures and, from the field theory perspective, this solution describes a deconfined thermal plasma that permeates the boundary. Notice that this was probably an unexpected result since the curvature of the hyperbolic space introduces a scale in the problem that could potentially result in a critical temperature for a phase transition between different solutions (this is what occurs for a field theory on $\mathbb{R} \times S^{2}$, where the radius of $S^{2}$ sets a scale that introduces a critical temperature where a confinement/deconfinement phase transition occurs [10]). The analysis of [42] was done only for uncharged hyperbolic black holes, but the main conclusions relevant for our purposes extend to the charged case.

In the case at hand the black droplets and funnels only approach the hyperbolic black hole in the asymptotic region $\chi \sim x_{0}$. Moreover, they also introduce a boundary black hole. This means that on the holographic boundary we have a boundary black hole in equilibrium with a thermal fluid or plasma that fills all the spacetime outside the black hole.

The difference between black funnels and black droplets is that, in the latter case, there are two disconnected event horizons in the bulk, one associated to the boundary black hole, and the other one associated to the asymptotic region of the boundary. The authors of [6] propose the interpretation that black funnels describe boundary black holes that couple strongly to the deconfined plasma. Under a thermal perturbation, heat flows easily from the boundary black hole towards the plasma and thermal equilibrium is rapidly achieved. In the bulk, such a perturbation flows continuously from the boundary black hole down the throat of the funnel and out of the shoulders of the funnel (described by the hyperbolic black hole dual to the deconfined plasma). On the other hand, the black droplet system describes a boundary black hole that couples only weakly to the deconfined plasma. Under a thermal perturbation it is now rather difficult for heat to flow from the boundary black hole to the plasma. In the bulk description this is because there is no connection between the droplet horizon (that reaches the boundary horizon) and the hyperbolic horizon deep in the bulk. As a consequence, the amount of heat that flows outward from the boundary black hole resembles the flow that occurs in a confined phase, even though the temperature is clearly much above any potential deconfinement/confinement transition [6]. From the field theory perspective, [6] interprets the weak coupling between the black hole and plasma as being due to the finite (and large) physical size of plasma excitations relative to the black hole size.

A closer inspection into the expectation value $\left\langle T_{a b}\right\rangle$ of the stress tensor given in (4.3) shows that the energy density $\left\langle T_{t t}\right\rangle$ of the quantum fields living on the boundary black hole background is positive in the asymptotic region, but becomes negative close to the event horizon. This mirrors the behavior one obtains from free field calculations of Hawking radiation, and it is precisely this violation of the classical energy condition that allows the 
black hole to bypass the area theorem and evaporate through emission of thermal radiation once backreaction is taken into account (see for example the reviews [43, 44]). We stress however that in our case no such backreaction occurs, because we have no propagating gravitational degree of freedom in the boundary theory. The results we obtain are not a 'semi-classical' approximation as in usual Hawking radiation computations, but the exact quantum result.

The quantum fields propagating on the boundary black hole spacetime behave effectively as a fluid, with stress energy tensor given precisely by (4.3). Due to the bulk gauge field, in addition to the background gravitational field described by the metric (2.14), there is also a background (non-dynamical) electromagnetic field $F_{a b}^{\text {bdy }}$, and the quantum fields generate a current $J^{a}$. By the AdS/CFT dictionary,

$$
F_{\mu \nu}^{\text {bdy }}=\lim _{r \rightarrow \infty} \gamma_{\mu}^{\rho} \gamma_{\nu}^{\sigma} F_{\rho \sigma}, \quad\left\langle J^{\mu}\right\rangle=\lim _{r \rightarrow \infty}\left(-\frac{\epsilon r}{\mathcal{A}}\right)^{3} \gamma^{\mu \nu} n^{\rho} F_{\nu \rho}
$$

yielding

$$
F^{\text {bdy }}=q_{e} \ell \mathrm{d} t \wedge \mathrm{d} \chi+\frac{q_{m}}{\mathcal{A}} \mathrm{d} \chi \wedge \mathrm{d} \phi, \quad\left\langle J^{a}\right\rangle=\left(-\frac{q_{e}}{\mathcal{A}}, 0, q_{m} \ell\right) .
$$

It can be verified that these quantities verify the magnetohydrodynamic equation

$$
D_{a}\left\langle T_{b}^{a}\right\rangle=\frac{1}{4 \pi \mathcal{G}} F_{b c}^{\mathrm{bdy}}\left\langle J^{c}\right\rangle,
$$

and the current conservation equation

$$
D_{a}\left\langle J^{a}\right\rangle=0
$$

as one expects on general grounds in AdS/CFT (here, $D_{a}$ is the boundary covariant derivative) [45-47]. It follows that the Lorentz force $F_{b c}^{\text {bdy }}\left\langle J^{c}\right\rangle$ on the fluid elements is always centrifugal. This force balances, together with the pressure of the fluid, the gravitational pull of the background geometry, leading to a stationary configuration. One peculiarity of the background geometry is that its constant time slices have finite volume, and so is the total charge carried by the fluid. Indeed, for the magnetic black droplet/funnel system $\left(q_{e}=0\right)$, the total magnetic charge (3.14) measured asymptotically can be rewritten, by construction, as

$$
\mathcal{Q}_{\text {bdy }}=\frac{1}{4 \pi} \int_{\mathcal{E}} F^{\text {bdy }},
$$

where the integral is performed over the region $\mathcal{E}$ external to the black hole on a constant $t$ surface. In three dimensions the flux (4.8) is by definition the total magnetic charge carried by the plasma. This can be seen for example by performing an electromagnetic duality in the bulk, that transforms the magnetic solution in an electric solution, with electric charge $q_{m}$. On the boundary, the transformation induces a duality in which the roles of $F^{\text {bdy }}$ and $\left\langle J^{\mu}\right\rangle$ are swapped, through a three-dimensional Hodge duality. The dualized fields are now

$$
\tilde{F}^{\text {bdy }}=*_{3}\left\langle J^{a}\right\rangle=q_{m} \ell \mathrm{d} t \wedge \mathrm{d} \chi+\frac{q_{e}}{\mathcal{A}} \mathrm{d} \chi \wedge \mathrm{d} \phi, \quad\left\langle\tilde{J}^{a}\right\rangle=*_{3} F^{\text {bdy }}=\left(\frac{q_{m}}{\mathcal{A}}, 0, q_{e} \ell\right)
$$


and it follows that the flux integral (4.8) can be recast as the volume integral of the magnetic charge density of the fluid,

$$
\mathcal{Q}_{\text {bdy }}=\frac{1}{4 \pi} \int_{\mathcal{E}} *_{3}\langle J\rangle=\frac{1}{4 \pi} \int_{\mathcal{E}}\left\langle\tilde{J}^{a}\right\rangle N_{a} \sqrt{\sigma} d^{2} \sigma
$$

where $N^{a}$ is the unit timelike normal to $\mathcal{E}$ in the boundary, and $\sqrt{\sigma} d^{2} \sigma$ its volume element. Therefore $\mathcal{Q}_{\text {bdy }}$ is precisely the total magnetic charge of the boundary plasma, i.e. the value of (4.10) agrees with (3.14).

In conclusion, the phase transition predicted by Hubeny et al. [5-7] for neutral boundary black holes does not occur when Maxwell fields are turned on, at least in the simple magnetically charged AdS C-metric system that we have analyzed in this article. The reason is, that in a black droplet system the magnetic charges redistribute among the black droplet and the planar black brane with charges of opposite sign on the two horizons. The resulting extra electromagnetic interaction, that favors the merging of the horizons, raises the free energy of the black droplet system up to the point that the black funnel always dominates the partition function.

Hence, in this simple toy model for strongly coupled Hawking radiation, and for the known solutions of the theory that are in thermal equilibrium, the black funnel always dominates the canonical ensemble. We stress however that other saddle points, possibly with lower free energy, might exist and that, more importantly, our argument does not exclude such a phase transition for strongly coupled fields propagating on other boundary black holes, a four-dimensional Schwarzschild background, for instance. This will be checked only once the gravitational dual of such a field theory is built, presumably resorting to numerical methods.

\section{Acknowledgments}

This article benefitted from several insightful discussions, that we warmly acknowledge, with Roberto Emparan, Veronica Hubeny, Don Marolf, Jiří Podolský, Mukund Rangamani, Harvey Reall and Simon Ross. MMC was supported in part by the FWO-Vlaanderen, project G.0235.05 and in part by the Federal Office for Scientific, Technical and Cultural Affairs through the "Interuniversity Attraction Poles Programme - Belgian Science Policy" P6/11-P. MMC acknowledges also support by the ANR grant STR-COSMO, ANR09-BLAN-0157, the ERC Advanced Grant 226371, the ITN programme PITN-GA-2009237920, the IFCPAR CEFIPRA programme 4104-2, the ANR programme NT09-573739 "string cosmo" and the PEPS-CNRS programme "Cordes, evolution, anisotropies et transitions". OJCD acknowledges financial support provided by the European Community through the Intra-European Marie Curie contract PIEF-GA-2008-220197. RM is funded by a postdoctoral fellowship of FNU-Denmark. This work was partially funded by FCTPortugal through project PTDC/FIS/099293/2008. 


\section{A The charged AdS C-metric: black droplets and black funnels}

In this appendix, we shall explore the full parameter space of the charged AdS Cmetric (2.1)-(2.3), and interpret the solutions in terms of black branes, black droplets and black funnels. To simplify the discussion, we shall define the total charge parameter $q$ to be

$$
q=\sqrt{q_{e}^{2}+q_{m}^{2}}
$$

and use, following $[5,6]$, the parameter $\lambda$ defined by

$$
\lambda=\frac{1}{\mathcal{A}^{2} \ell^{2}}-1,
$$

in place of the acceleration parameter $\mathcal{A}$. Then, the solutions with negative cosmological constant we are interested in correspond to the $\lambda>-1$ range.

As explained in section 2.1, the asymptotic region of the manifold is located on the $x=y$ hypersurface, and the physical properties of the solutions are determined by the polynomials $F(\xi)$ and $G(\xi)$. In particular, roots of $F$ correspond to event horizons, and the zeroes of $G$ signal the presence of axes of symmetry of the spacetime. Asymptotic regions on the boundary $x=y$ are located at double (or triple) roots of $G$. If such degenerate roots are absent, the boundary is compact. In coincidence of these axes, we have a conical singularity unless the periodicity of the angular coordinate $\phi$ is set according to (2.5). If two such single roots are visible from a single static region (that is in the cases of a single black droplet, of a single planar black hole or of a black droplet floating on a planar black hole), there is a potential conical singularity, meaning that at least one of the horizons is accelerated by a cosmic string stretching from it to infinity or to the other horizon, unless both singularities can be contemporaneously eliminated by a single choice of period for $\phi$, i.e. $\left|G^{\prime}\left(x_{i} \mid\right)=\right| G^{\prime}\left(x_{j}\right) \mid$. Finally, the temperature of the event horizon at the root $y_{i}$ of the function $F(y)$ is obtained by requiring that no conical singularity appears in the Euclidean continuation of the manifold, and is given by (as explained in section 2.1)

$$
T_{i}=\frac{1}{\beta_{i}}=\frac{\left|F^{\prime}\left(y_{i}\right)\right|}{4 \pi \sqrt{1+\lambda}} .
$$

Again, when in presence of more than one horizon, a single choice of periodicity for the Euclidean time will not generally be sufficient to eliminate all conical singularities, and thermal equilibrium will be realized only for special cases.

Here, we want to classify the C-metric spacetimes according to their physical content in terms of black objects, and to this end we need to study parametrically the behavior of these polynomials, and then use the information on their roots to interpret the resulting spacetimes.

More precisely we are interested, given some static boundary conditions, in the static region of the bulk solution. It can present one or two event horizon; we will classify them according to the behavior of the $\partial_{\phi}$ axes/singularities, following the definition proposed in [6]. Since outer horizons lie at constant $y$, they intersect the $\partial_{\phi}$ axes or singularities (that are at constant $x$ ) once or twice. If the horizon has two such intersections, it will 


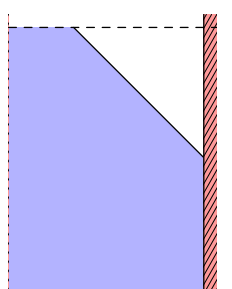

Black funnel

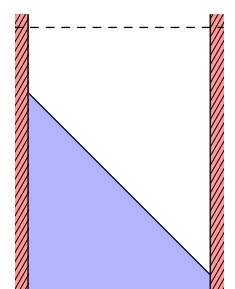

Planar black hole

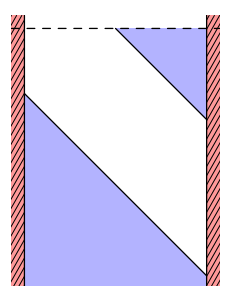

Black droplet on a planar bh

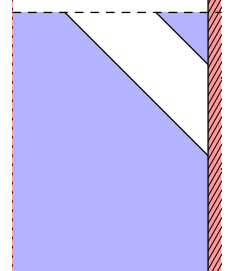

Black droplet on a black funnel

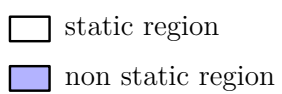

$-\rightarrow$ AdS boundary

$\triangle$ event horizon

Figure 8. Interpretation of the solutions: possible kinds of static regions that can be seen from a static boundary region, interpreted in terms of black droplets, black funnels, and planar black holes. The planar black hole and the black droplet floating over a planar black hole cases have two zeros of the function $G$ in the static region, and therefore suffer in general of a conical singularity. Other cases can arise where one of the $x$ constant boundaries is a double or triple root of $G$, in which case it corresponds to an asymptotic region of the boundary, and black funnels and black droplets with degenerate horizons.

obviously not reach the asymptotic boundary: such black holes are referred to as planar black holes, irrespectively of their geometry or topology. If the horizon intersects a single axis/singularity, two situations can arise; if the axis connects the horizon to the boundary through the external region, the horizon is called a black funnel, while if the connection occurs in its interior, it is dubbed black droplet.

To identify the black hole content of a particular solution, determined by a choice of physical parameters, it is convenient to represent on the $(x, z)$ plane (with $z=y-x$ ) the roots $y=y_{i}$ corresponding to the horizons, and the roots $x=x_{i}$ corresponding to the axes (or asymptotic regions of the boundary if they are degenerate; in that case we represent them with double lines). An example, corresponding to the thermal equilibrium configuration with an asymptotic region studied in this article, is given in figure 4 . One then determines the static and non-static regions, that are separated by the $y_{i}$ roots; in our diagram we shaded the non-static regions. Then, each connected white region corresponds to a single static solution of the bulk gravitational equations. The dashed horizontal line at $x=y$ represents the boundary of the asymptotically AdS spacetime. If two different static regions share the same boundary at $x=y$, then they represent two competing gravitational saddle points dual to the same field theory state. Then, the interpretation of the horizons present in the solution according to the definition given in [6], is easily obtained according to figure 8. The interested reader can find more details on the procedure in [6].

We will consider the three cases $\kappa=0, \pm 1$ separately.

$\boldsymbol{\kappa}=\mathbf{- 1}$, hyperbolic horizons. The function $G(\xi)$ takes in this case the form $G(\xi)=$ $1+\xi^{2}-2 \mu \xi^{3}-q^{2} \xi^{4}$, whose derivative has exactly three single real roots, in $\xi=0$ and in

$$
\tilde{x}_{0,1}=-\frac{1}{\sqrt{2}} \frac{q_{\star}}{q^{2}}\left(1 \pm \sqrt{1+\frac{q^{2}}{q_{\star}^{2}}}\right),
$$


then $\tilde{x}_{0}<0<\tilde{x}_{1}$, and $G$ has local maxima in $\tilde{x}_{0,1}$ and a minimum in the origin. It is furthermore easy to show that $G\left(\tilde{x}_{0}\right) \geq G\left(\tilde{x}_{1}\right)>1$ with the equality holding only for $\mu=0$. Therefore $G$ has precisely two single real roots $x_{0}$ and $x_{1}$, such that $x_{0}<\tilde{x}_{0}<$ $0<\tilde{x}_{1}<x_{1}$. As we shall see, the root structure is the same as that arising in the $\kappa=+1$ III.A. case. Varying $\lambda$, the roots merge and split, leading to static regions with black funnels/droplet/branes and singularities, that can easily be recognized by drawing the corresponding $x$ - $z$ diagrams.

$\boldsymbol{\kappa}=\mathbf{0}$, flat planar horizons. The function $G(y)$ has a maximum in $\tilde{y}_{0}=-\frac{3 \mu}{2 q^{2}}<0$ and an inflection point in $\tilde{y}_{1}=0$ corresponding to a double root of its derivative. $G$ has therefore two real roots $y_{0}$ and $y_{1}$, satisfying the relation $y_{0}<\tilde{y}_{0}<0<y_{1}$. Hence, the function $F(\xi)$, obtained by a reflection of $G(\xi)$ with respect to the $\xi$ axis, followed by a translation, has a minimum in $\tilde{y}_{0}$, and zeroes if $F\left(\tilde{y}_{0}\right) \leq 0$. Hence, defining

$$
\lambda_{\star}=\frac{27 \mu^{4}}{16 q^{6}},
$$

$F$ has two real roots $x_{0}$ and $x_{1}$ for $-1<\lambda<\lambda_{\star}$, one double root $x_{0}=x_{1}$ for $\lambda=\lambda_{\star}$ and no roots if $\lambda>\lambda_{\star}$. The root structure is the same as for cases $\kappa=+1$ I, II.A, III.D (with $x_{2}$ assuming the role of $x_{0}$ ) and III.E, that we shall study shortly. The static region has a conical singularity for $\lambda \geq \lambda_{\star}$, otherwise it represents a regular black funnel or a black droplet with a naked singularity.

$\kappa=+\mathbf{1}$, spherical horizons. The derivative of the function $G(\xi)$ is now

$$
G^{\prime}(\xi)=-4 q^{2} \xi P(\xi), \quad P(\xi)=\xi^{2}+\frac{3 \mu^{2}}{2 q^{2}} \xi+\frac{1}{2 q^{2}},
$$

and the behavior of the second-order polynomial $P(x)$ is determined by the relative value of $q$ with respect to the critical charge

$$
q_{\star}=\frac{3 \mu}{2 \sqrt{2}} .
$$

In particular $P(\xi)$ has no real roots for $q>q_{\star}$, one double root $\tilde{x}=-2 / 3 \mu$ for $q=q_{\star}$, and two negative roots $\tilde{x}_{0}<\tilde{x}_{1}<0$ for $q<q_{\star}$.

I. $q>q_{\star}$

In this case $P(\xi)>0$ and $G^{\prime}(\xi)$ has a single root in $\xi=0$, where $G(\xi)$ assumes its maximum. Therefore, $G(\xi) \leq 1$ and $F(y)$ has two single real roots $\tilde{y}_{0}<0<\tilde{y}_{1}$ for $-1<\lambda<0$, one double real root in $\tilde{y}_{0}=0$ for $\lambda=0$ and no real roots for $\lambda>0$. Again, with the same root structure as in the $\kappa=0$ case, we have conical singularities for $\lambda \geq 0$, and otherwise black funnels or a black droplet with a naked singularity.

II. $q=q_{\star}$

$P$ has a double root in $\tilde{x}=-2 / 3 \mu$, where $G(x)$ has an inflection point. Therefore, the function $G$ has precisely two roots $x_{0}$ and $x_{1}$. If we define the critical mass $\mu_{\star}=\sqrt{2} / 3 \sqrt{3}$, then we have the following cases: 
II.A. $q_{\star} \neq \frac{1}{2 \sqrt{3}}\left(\mu \neq \mu_{\star}\right)$

then both real roots $x_{1}$ and $x_{2}$ are real roots, and they satisfy $x_{0}<\tilde{x}<0<x_{1}$ for $\mu>\mu_{\star}$ and $\tilde{x}<x_{0}<0<x_{1}$ for $\mu<\mu_{\star}$. The behavior is hence the same as the previous case I and as the $\kappa=0$ one with conical singularities and singular black droplets, apart from a very special case discussed below $\left(\mu<\mu_{\star}\right.$ and $\left.\lambda=\lambda_{\star}\right)$.

II.B. $q_{\star}=\frac{1}{2 \sqrt{3}}\left(\mu=\mu_{\star}\right)$

In the critical case $\mu=\mu_{\star}$, the smaller root of $G, x_{0}=\tilde{x}=-\sqrt{6}$ becomes a triple root, and the polynomial becomes

$$
G(x)=-\frac{1}{12}\left(x-\sqrt{\frac{2}{3}}\right)(x+\sqrt{6})^{3} .
$$

Now the solution develops a new asymptotic region on the boundary in correspondence of the first axis that gets pushed infinitely far away from any other point in the manifold, while the second axis $x_{3}$ is smooth as we choose the associated periodicity for the angle $\varphi$. The boundary field theory states are therefore dual to two competing gravitational saddle points, one representing a regular black funnel, the other a single black droplet. Unfortunately, the black droplet has a curvature singularity in $y \rightarrow \infty$ that is not clothed by any horizon.

Since $G(x) \leq 1$ for any $x, F$ has no roots for $\lambda>0$, and a double root $y_{0}=y_{1}=0$ for $\lambda=0$. Finally, if $-1<\lambda<0$ the polynomial $F$ has two real roots $y_{0}, y_{1}$ satisfying $x_{0}<y_{0}<0<y_{1}<x_{1}$. These roots are always single roots for $\mu \geq \mu_{\star}$, but when $\mu<\mu_{\star}$ the smaller root $y_{0}$ becomes a triple root for the critical value $\lambda=\lambda_{\star}=-\mu_{\star}^{2} / \mu^{2}=-1 / 12 q_{\star}^{2}$. The different cases are therefore:

- $\lambda>0$ : No roots of $F$ (no horizons) $x_{0}<0<x_{1}$

- $\lambda=0$ : One double root of $F$ in $y=0, x_{0}<y_{0}=y_{1}=0<x_{1}$

- $-1<\lambda<0$ : Two roots $y_{0,1}$ of $F, x_{0}<y_{0}<0<y_{1}<x_{1}$. $y_{1}$ is a single root, $y_{0}$ is a triple root for $\mu<\mu_{\star}, \lambda=\lambda_{\star}$, and a single root otherwise.

III. $q<q_{\star}$

In this case $P(\xi)$ has two single real roots $\tilde{x}_{0}<\tilde{x}_{1}<0$, in correspondence of which the function $G(x)$ assumes a maximum and a minimum respectively. If $q>1 / 2 \sqrt{3}$, both $G\left(\tilde{x}_{0}\right)$ and $G\left(\tilde{x}_{1}\right)$ are positive, and $G(x)$ has only two single roots. If $q<1 / 2 \sqrt{3}$, define

$$
q_{\star}^{ \pm}(q)=\frac{1}{4 \sqrt{3}} \sqrt{1+36 q^{2} \pm\left(1-12 q^{2}\right)^{\frac{3}{2}}},
$$

such that $G\left(\tilde{x}_{0}\right)=0$ for $q_{\star}^{-}=q_{\star}$ and $G\left(\tilde{x}_{1}\right)=0$ for $q_{\star}^{+}=q_{\star}$. Then, we have the following cases

III.A. $q_{\star}>1 / 2 \sqrt{3}$ or $q_{\star}>q_{\star}^{+}$

both $G\left(\tilde{x}_{0}\right)$ and $G\left(\tilde{x}_{1}\right)$ are positive, and $G$ has two single real roots. We are therefore qualitatively in the same situation as that described in the $\kappa=-1$ case. 

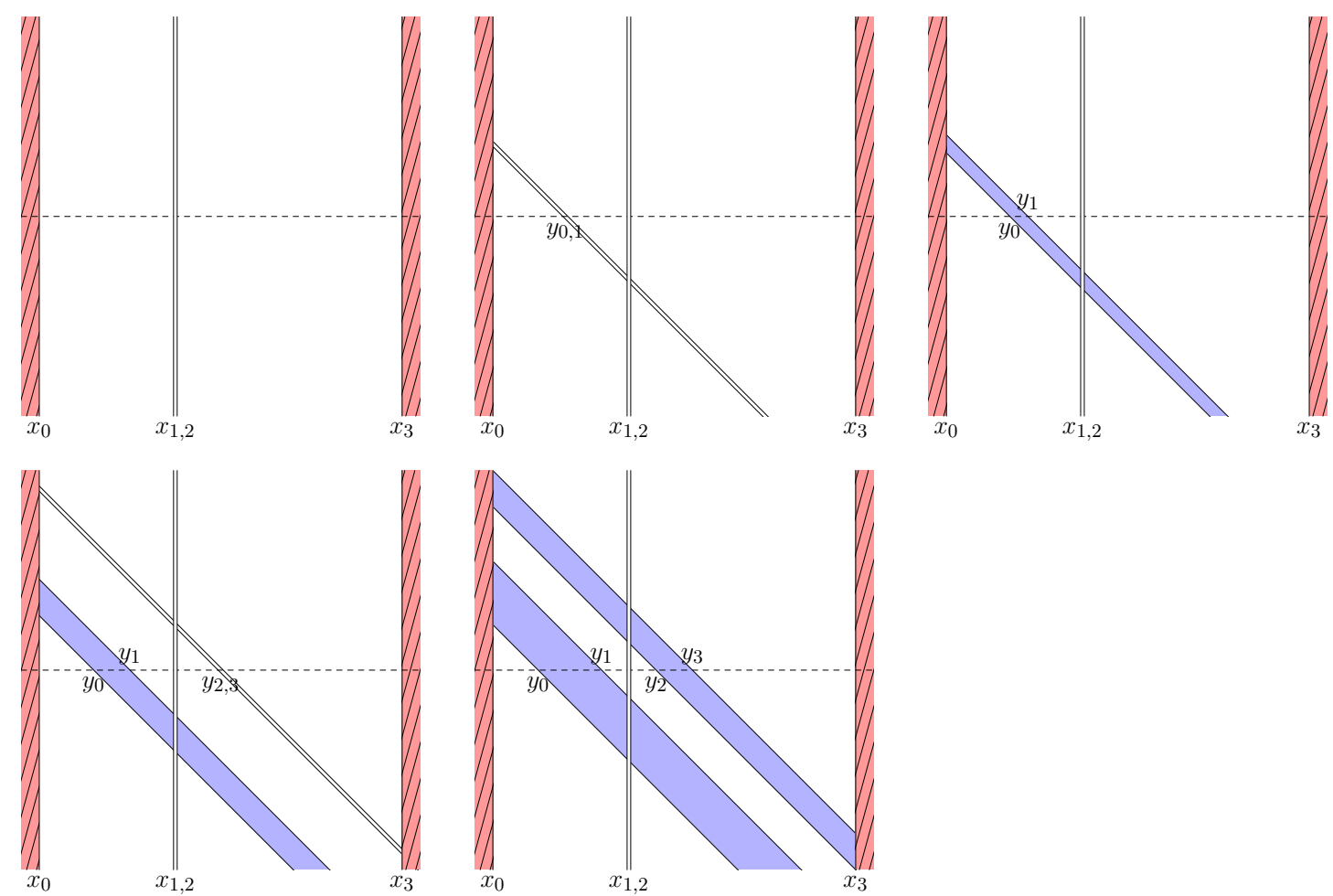

Figure 9. Diagram of the $z-x$ plane corresponding to the case III.B. The double root $x_{1}=x_{2}$ is the asymptotic region, and its intersection with the $z=0$ dashed line represents the asymptotic region of the boundary.

III.B. $q_{\star}=q_{\star}^{+}$

$\tilde{x}_{1}$ is a double root of $G$, hence $G$ has two three real roots $x_{0}<x_{1}<0<x_{2}$, with $x_{0}$ and $x_{2}$ single roots and $x_{1}=\tilde{x}_{1}$ double root. Note that in correspondence of the double root of $G$ there is an asymptotic region, and its intersection with the $z=0$ boundary gives the asymptotic region of the boundary. The solutions in thermal equilibrium on which we focus in this paper belong to this class, for special parameters. For illustrative purposes, we show all the diagrams in the $z$-x plane in figure 9 for this class of solutions. On them, one can observe the evolution of the horizons as the parameter $\lambda$ is varied. Similar behavior is obtained for the other cases, although we choose not to show all the diagrams to keep the presentation short.

III.C. $q_{\star}^{-}<q_{\star}<q_{\star}^{+}$

the local minimum in $\tilde{x}_{1}$ is negative, while the maximum in $\tilde{x}_{1}$ is positive, hence $G$ has four single real roots $x_{0}<\tilde{x}_{0}<x_{1}<\tilde{x}_{1}<x_{2}<0<x_{3}$. Now the spacetime has axes of symmetries in both $x_{1}$ and $x_{2}$, and exhibits generally a conical singularity that cannot be eliminated.

III.D. $q_{\star}=q_{\star}^{-}$

now $\tilde{x}_{0}$ is a double root of $G$, and we have three roots $x_{0}=\tilde{x}_{0}<x_{1}<0<x_{2}$, with $x_{1,2}$ single roots and $x_{0}$ double root. Since the double root appears in a 


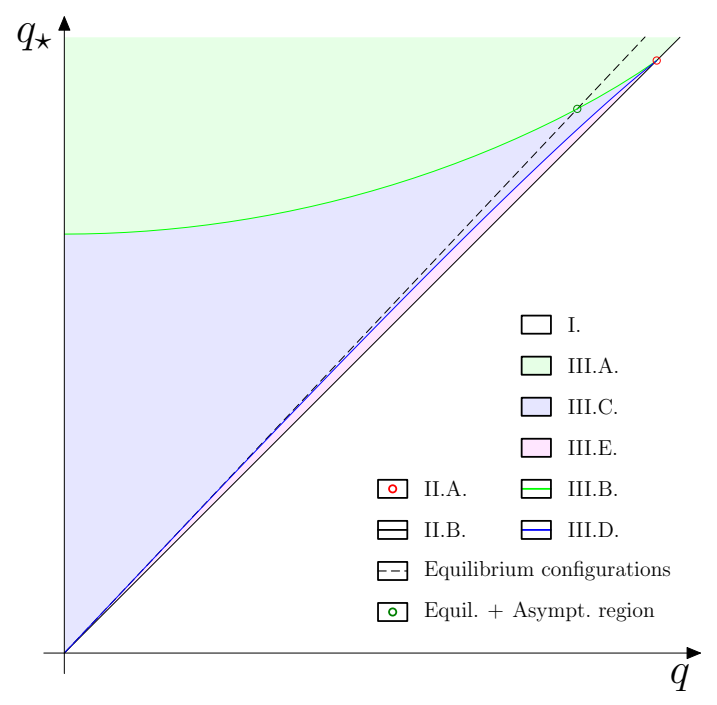

Figure 10. Summary for the $\kappa=+1$ case.

region with wrong signature, it is not relevant for us and the allowed range of the $x$ coordinates is simply $\left(x_{2}, x_{3}\right)$. The resulting $z-x$ diagrams are therefore the ones already described in the $\kappa=0$ case, where now $x_{2}$ has the role of $x_{0}$.

III.E. $q<q_{\star}<q_{\star}^{-}$

both local extrema of $\mathrm{G}$ in $\tilde{x}_{0,1}$ are negative, therefore $G$ has only two single real roots in $x_{0}<0<x_{1}$. The situation is again analogous to the one described in the $\kappa=0$ case.

We will not enter in the details of what happens as $\lambda$ is varied, as this can be easily determined case by case, but the situation can be summed up by saying that the roots $y_{2}$ and $y_{3}$ of $F$ are always present for $-1<\lambda<0$, they merge in a double root for $\lambda=0$ and they disappear for $\lambda>0$. The roots $y_{0}$ and $y_{1}$ exist as long as $\lambda<G\left(\tilde{x}_{0}\right)-1$. For the critical value $\lambda_{\star}=G\left(\tilde{x}_{0}\right)-1$, they merge in a double root, and for $\lambda>\lambda_{\star}$ they disappear.

In the case III.A., in addition of the previous behavior of the roots of $F$, one has that for $\lambda=G\left(\tilde{x}_{1}\right)-1$ the roots $y_{1}$ and $y_{2}$ merge, and they disappear for lower values of $\lambda$.

The different behaviors of the polynomial $G$ have been summarized in figure 10 in the parameter space $q_{\star^{-}} q$ (or equivalently the $\mu-q$ plane).

\section{B Configurations in thermal equilibrium}

As we discussed in the previous section, the C-metric presents an event horizon at each root $y_{i}$ of the function $F(y)$, whose temperature is given by equation (A.3). It follows that generically, solutions with more than one event horizons are not in thermal equilibrium because the corresponding black holes are at different temperatures. In particular, black droplets floating over planar branes (or over black funnels) have different temperatures 
and, taking into account quantum corrections, we cannot expect them to be true stationary equilibrium configurations; the evolution should drive them through quantum evaporation to the funnel solution with the same temperature as the initial black droplet solution (fixed by the boundary conditions). It is therefore interesting to look whether such equilibrium configurations can exist. To this end, we parameterize the polynomial $F(y)$ using its roots $y_{0}, \ldots, y_{3}$, and assume that at least $y_{1}<y_{2}$ are distinct real roots. Then

$$
F(y)=q^{2} \prod_{i=0}^{3}\left(y-y_{i}\right)=q^{2}\left(y^{4}-\sigma y^{3}+t y^{2}-\rho h y+\rho\right)
$$

with

$$
\sigma=\sum y_{i}=-\frac{2 \mu}{q^{2}}, \quad \rho=\prod y_{i}=\frac{\kappa}{q^{2}}, \quad h=\sum \frac{1}{y_{i}}=0, \quad t=\sum_{i<j} y_{i} y_{j}=\frac{\lambda}{q^{2}} .
$$

The equilibrium requirement that the horizons in $y_{0}$ and $y_{1}$ have the same temperature is then $\left|F^{\prime}\left(y_{1}\right)\right|=\left|F^{\prime}\left(y_{2}\right)\right|$; however if $F^{\prime}$ has the same sign on both roots, there must be a third root in between, and $y_{1}, y_{2}$ are not separated by a static region. We will therefore look for values of the parameters such that $F^{\prime}\left(y_{1}\right)=-F^{\prime}\left(y_{2}\right)$. This condition implies that the other two roots are real and satisfy $y_{0}+y_{3}=y_{1}+y_{2}$ and $y_{0} y_{3}=-y_{1} y_{2}$, which can be solved to give

$$
y_{0,3}=\frac{1}{2}\left(y_{1}+y_{2} \mp \sqrt{\left(y_{1}+y_{2}\right)^{2}+4 y_{1} y_{2}}\right) .
$$

Therefore, configurations in thermodynamic equilibrium always have four horizons, ordered according to $y_{0}<y_{1}<y_{2}<y_{3}$. Denoting $y_{1}=-a$ and $y_{2}=-b$, the polynomial can be rewritten in the form

$$
F(y)=-\left(\frac{a b}{a+b}\right)^{2}\left(1+\frac{y}{a}\right)\left(1+\frac{y}{b}\right)\left(1-\frac{a+b}{a b} y-\frac{1}{a b} y^{2}\right) .
$$

Then, $\kappa=q^{2}(a+b)^{2}>0$ and therefore we are in the $\kappa=+1$ case, and the physical parameters can be written parametrically in terms of $a, b$ as

$$
\mu=q=\frac{1}{a+b}, \quad \lambda=\frac{1}{\ell^{2} \mathcal{A}^{2}}-1=-\left(\frac{a b}{a+b}\right)^{2},
$$

with the restriction $a+b>0$ coming from positivity of $\mu$ and $q$. The critical charge reads then $q_{\star}=3 q / 2 \sqrt{2}$. We are therefore in the case $\kappa=+1$, III $\left(q<q_{\star}\right)$ of our classification, with $q_{\star}>q_{\star}^{-}$as can be readily checked; and the value of $\mu=q$ fully determines the black objects content of the solution, as follows:

- $\mu=q>1 / 4$ : Case III.A, we have a black funnel at thermal equilibrium with a black droplet floating over it. The asymptotic boundary is conformally compact and the static region of the metric is regular.

- $\mu=q=1 / 4$ : Case III.B, the condition implies $a=4-b$, and results in the one parameter family of solutions given explicitely in equation (2.9). The boundary is 

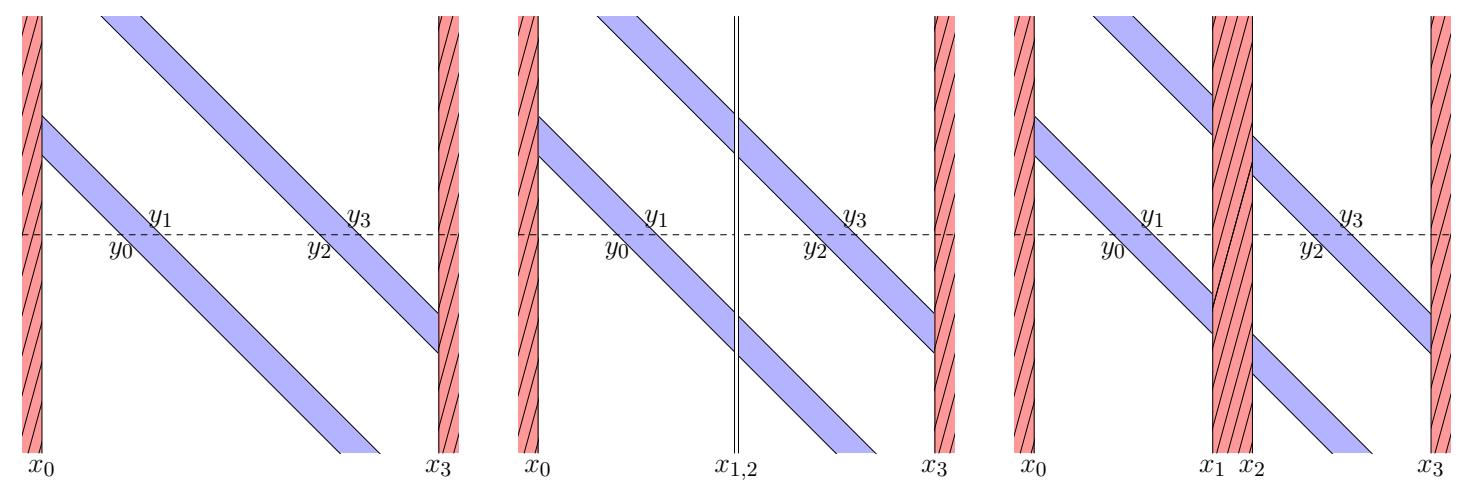

Figure 11. Equilibrium configurations with $\kappa=+1, \mu=q$ and $\lambda<0$. The horizons share the same temperature, and the spacetime is therefore at thermal equilibrium. From left to right, a black droplet floating over a black funnel for $\mu>1 / 4$, a black droplet floating on a black hole with an asymptotic region for $\mu=1 / 4$, and a black droplet and a planar black hole with a conical singularity for $\mu<1 / 4$.

not compact and has an asymptotic region, the solution represents a planar black hole at equilibrium with a black droplet floating over it in one sector, and a black funnel in the other. This is the regular solution that was analysed in detail in sections 2 and 3 .

- $\mu=q<1 / 4$ : Case III.C, again a planar black hole at equilibrium with a black droplet floating over it, but with a conformally compact boundary. Potentially a conical singularity could occur in this case, but a simple check shows that they cannot be eliminated simultaneously on both axis if $0<\mu<1 / 4$ and therefore this case is singular.

Indeed, the roots of the function $G(x)$ that signals the axes of symmetry are given explicitely by

$$
x_{0}=\frac{-1-\sqrt{1+4 \mu}}{2 \mu}, \quad x_{1}=\frac{-1-\sqrt{1-4 \mu}}{2 \mu}, \quad x_{2}=\frac{-1+\sqrt{1-4 \mu}}{2 \mu}, \quad x_{3}=\frac{-1+\sqrt{1+4 \mu}}{2 \mu} .
$$

The roots $x_{1}$ and $x_{2}$ do not exist for $\mu>1 / 4$, for $\mu=1 / 4$ they degenerate in a double real root corresponding to the asymptotic region of the boundary, and for $0<\mu<1 / 4$ we have four distinct single real roots $x_{0}<x_{1}<x_{2}<x_{3}$. It is then trivial to check that the relation $G^{\prime}\left(x_{2}\right)=-G^{\prime}\left(x_{3}\right)$ (or $G^{\prime}\left(x_{0}\right)=-G^{\prime}\left(x_{1}\right)$ ) cannot be satisfied for any choice of $0<\mu<1 / 4$, meaning that a conical singularity is unavoidable.

If $\lambda=0$, the two horizons are degenerate, corresponding to double roots of $F$, and the temperature of the system vanish. For $-1<\lambda<0$, the horizons have finite temperature and the solutions correspond to systems of two horizons at thermal equilibrium. The corresponding $z-x$ diagrams can be found in figure 11 .

These equilibrium configurations are plotted with the dashed lines in figure 10. The first two cases have genuine regular external regions at thermal equilibrium, the latter having been investigated thoroughly in the body of this article. The reader might wonder whether a similar analysis, involving two geometries competing to minimize the free energy 


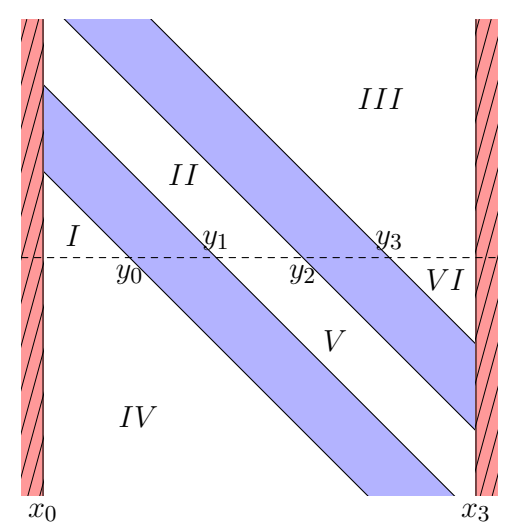

Figure 12. Thermal equilibrium configuration with $\kappa=+1$ and $\mu=q>1 / 4$, case III.A. The regions $I I$ and $V$ share the same boundary, but they are related by an isometry of the C-metric.

could be performed in the first case. Before trying to answer this question, we need however to establish a symmetry of those solutions.

A symmetry relating configurations in thermal equilibrium: the requirement that the temperatures of the horizons at $y_{0}$ and at $y_{1}$ coincide imposes that the derivatives of the polynomials $F$ and $G$ in those two points are opposite and the tangents to these curves are reflected through a vertical axis. This in turn forces the full polynomials to be symmetric under that same reflection, and induces a discrete isometry on the C-metric. Indeed, if one shifts the coordinates $x$ and $y$ according to

$$
\tilde{x}=x+\frac{1}{2 \mu}, \quad \tilde{y}=y+\frac{1}{2 \mu},
$$

the metric (2.1) remains invariant in form since $\tilde{x}-\tilde{y}=x-y$. The $F$ and $G$ polynomials get transformed into

$$
\begin{aligned}
& \tilde{F}(\tilde{y})=F\left(\tilde{y}-\frac{1}{2 \mu}\right)=\frac{1}{(a+b)^{2}}\left[\tilde{y}^{2}-\left(\frac{a-b}{2}\right)^{2}\right]\left[\tilde{y}^{2}-\frac{1}{4}(a+b)^{2}-a b\right], \\
& \tilde{G}(\tilde{x})=G\left(\tilde{x}-\frac{1}{2 \mu}\right)=-\frac{\tilde{x}^{4}}{(a+b)^{2}}+\frac{1}{2} \tilde{x}^{2}+\frac{2}{\mathcal{A}^{2} \ell^{2}}-\frac{(a-b)^{2}}{16(a+b)^{2}} .
\end{aligned}
$$

These polynomials are even, and therefore the full metric (2.1) is invariant left invariant under the transformation

$$
\tilde{x} \mapsto-\tilde{x}, \quad \tilde{y} \mapsto-\tilde{y} .
$$

On the other hand, the gauge potential transforms as $A \mapsto A$, and the electric charge and magnetic charge get reversed under this transformation, $q_{e} \mapsto-q_{e}$ and $q_{m} \mapsto-q_{m}$. This isometry acts therefore on the $\mu=q=1 / 4$ solution by exchanging regions $I$ and $I I I$ with regions $I V$ and $I I$ respectively in diagram of figure 4 respectively, and inverting the charges; we conclude that region $I$ is isometric to region $I V$ and region $I I$ is isometric to region $I I I$, as advertised in section 2.2, and (up to the sign of the charges) the results obtained for quadrant $I(I I I)$ are valid also for quadrant $I V(I I)$.

We finally can consider the $\mu=q>1 / 4$. The corresponding diagram is shown in figure 12, and has six distinct static external regions. Of those, only regions $I I$ and $V$ are 
regular, but as they share the same asymptotic boundary, one might wonder which one dominates the partition function of the dual CFT. Again, the isometry (B.10) comes to our help since it maps region $I I$ to region $V$, and therefore these regions do not represent two distinct gravitational instantons in competition to minimize the free energy, and no black funnel/black droplet transition is present in this case.

In conclusion, the $\mu=q=1 / 4$ case studied in this article is the only one in which black droplets and black funnels at thermal equilibrium compete to dominate the free energy among the solutions of the charged AdS C-metrics.

Open Access. This article is distributed under the terms of the Creative Commons Attribution Noncommercial License which permits any noncommercial use, distribution, and reproduction in any medium, provided the original author(s) and source are credited.

\section{References}

[1] S.W. Hawking, Black hole explosions, Nature 248 (1974) 30 [SPIRES].

[2] S.W. Hawking, Particle creation by black holes, Commun. Math. Phys. 43 (1975) 199 [Erratum ibid. 46 (1976) 206] [SPIRES].

[3] N.D. Birrell and P.C.W. Davies, Quantum fields in curved space, Cambridge University Press, Cambridge U.K. (1982) [SPIRES].

[4] S.F. Ross, Black hole thermodynamics, hep-th/0502195 [SPIRES].

[5] V.E. Hubeny, D. Marolf and M. Rangamani, Hawking radiation in large- $N$ strongly-coupled field theories, Class. Quant. Grav. 27 (2010) 095015 [arXiv:0908.2270] [SPIRES].

[6] V.E. Hubeny, D. Marolf and M. Rangamani, Black funnels and droplets from the AdS C-metrics, Class. Quant. Grav. 27 (2010) 025001 [arXiv:0909.0005] [SPIRES].

[7] V.E. Hubeny, D. Marolf and M. Rangamani, Hawking radiation from AdS black holes, Class. Quant. Grav. 27 (2010) 095018 [arXiv: 0911.4144] [SPIRES].

[8] J.M. Maldacena, The large- $N$ limit of superconformal field theories and supergravity, Int. J. Theor. Phys. 38 (1999) 1113 [Adv. Theor. Math. Phys. 2 (1998) 231] [hep-th/9711200] [SPIRES].

[9] S.S. Gubser, I.R. Klebanov and A.M. Polyakov, Gauge theory correlators from non-critical string theory, Phys. Lett. B 428 (1998) 105 [hep-th/9802109] [SPIRES].

[10] E. Witten, Anti-de Sitter space and holography, Adv. Theor. Math. Phys. 2 (1998) 253 [hep-th/9802150] [SPIRES].

[11] J.F. Plebanski and M. Demianski, Rotating, charged and uniformly accelerating mass in general relativity, Ann. Phys. 98 (1976) 98 [SPIRES].

[12] J. Podolsky, Accelerating black holes in anti-de Sitter universe, Czech. J. Phys. 52 (2002) 1 [gr-qc/0202033] [SPIRES].

[13] O.J.C. Dias and J.P.S. Lemos, Pair of accelerated black holes in anti-de Sitter background: the AdS C-metric, Phys. Rev. D 67 (2003) 064001 [hep-th/0210065] [SPIRES].

[14] P. Krtous, Accelerated black holes in an anti-de Sitter universe, Phys. Rev. D 72 (2005) 124019 [gr-qc/0510101] [SPIRES]. 
[15] F. Mellor and I. Moss, Black holes and quantum wormholes, Phys. Lett. B 222 (1989) 361 [SPIRES].

[16] F. Mellor and I. Moss, Black holes and gravitational instantons, Class. Quant. Grav. 6 (1989) 1379 [SPIRES].

[17] L.J. Romans, Supersymmetric, cold and lukewarm black holes in cosmological Einstein-Maxwell theory, Nucl. Phys. B 383 (1992) 395 [hep-th/9203018] [SPIRES].

[18] R.B. Mann and S.F. Ross, Cosmological production of charged black hole pairs, Phys. Rev. D 52 (1995) 2254 [gr-qc/9504015] [SPIRES].

[19] I.S. Booth and R.B. Mann, Cosmological pair production of charged and rotating black holes, Nucl. Phys. B 539 (1999) 267 [gr-qc/9806056] [SPIRES].

[20] O.J.C. Dias and J.P.S. Lemos, Pair creation of de Sitter black holes on a cosmic string background, Phys. Rev. D 69 (2004) 084006 [hep-th/0310068] [SPIRES].

[21] V. Cardoso, O.J.C. Dias and J.P.S. Lemos, Nariai, Bertotti-Robinson and anti-Nariai solutions in higher dimensions, Phys. Rev. D 70 (2004) 024002 [hep-th/0401192] [SPIRES].

[22] O.J.C. Dias and J.P.S. Lemos, The extremal limits of the C-metric: Nariai, Bertotti-Robinson and anti-Nariai C-metrics, Phys. Rev. D 68 (2003) 104010 [hep-th/0306194] [SPIRES].

[23] O.J.C. Dias, Pair creation of anti-de Sitter black holes on a cosmic string background, Phys. Rev. D 70 (2004) 024007 [hep-th/0401069] [SPIRES].

[24] R.B. Mann, Pair production of topological anti-de Sitter black holes, Class. Quant. Grav. 14 (1997) L109 [gr-qc/9607071] [SPIRES].

[25] R. Emparan, G.T. Horowitz and R.C. Myers, Exact description of black holes on branes, JHEP 01 (2000) 007 [hep-th/9911043] [SPIRES].

[26] R. Emparan, G.T. Horowitz and R.C. Myers, Exact description of black holes on branes. II: comparison with BTZ black holes and black strings, JHEP 01 (2000) 021 [hep-th/9912135] [SPIRES].

[27] C. Charmousis and R. Gregory, Axisymmetric metrics in arbitrary dimensions, Class. Quant. Grav. 21 (2004) 527 [gr-qc/0306069] [SPIRES].

[28] J. Podolsky, M. Ortaggio and P. Krtous, Radiation from accelerated black holes in an anti-de Sitter universe, Phys. Rev. D 68 (2003) 124004 [gr-qc/0307108] [SPIRES].

[29] M. Rogatko, Cosmological black holes on branes, Phys. Rev. D 69 (2004) 044022 [hep-th/0312105] [SPIRES].

[30] J. Podolsky and J.B. Griffiths, Accelerating Kerr-Newman black holes in (anti-)de Sitter space-time, Phys. Rev. D 73 (2006) 044018 [gr-qc/0601130] [SPIRES].

[31] R. Emparan and G. Milanesi, Exact gravitational dual of a plasma ball, JHEP 08 (2009) 012 [arXiv:0905.4590] [SPIRES].

[32] C. Charmousis, T. Kolyvaris and E. Papantonopoulos, Charged C-metric with conformally coupled scalar field, Class. Quant. Grav. 26 (2009) 175012 [arXiv:0906.5568] [SPIRES].

[33] A. Anabalon and H. Maeda, New charged black holes with conformal scalar hair, Phys. Rev. D 81 (2010) 041501 [arXiv:0907.0219] [SPIRES]. 
[34] J.B. Griffiths and J. Podolsky, Exact space-times in Einstein's general relativity, Cambridge University Press, Cambridge U.K. (2009).

[35] R. Emparan, Rotating circular strings and infinite non-uniqueness of black rings, JHEP 03 (2004) 064 [hep-th/0402149] [SPIRES].

[36] M. Henningson and K. Skenderis, The holographic Weyl anomaly, JHEP 07 (1998) 023 [hep-th/9806087] [SPIRES].

[37] M. Henningson and K. Skenderis, Holography and the Weyl anomaly, Fortsch. Phys. 48 (2000) 125 [hep-th/9812032] [SPIRES].

[38] V. Balasubramanian and P. Kraus, A stress tensor for anti-de Sitter gravity, Commun. Math. Phys. 208 (1999) 413 [hep-th/9902121] [SPIRES].

[39] R. Emparan, C.V. Johnson and R.C. Myers, Surface terms as counterterms in the AdS/CFT correspondence, Phys. Rev. D 60 (1999) 104001 [hep-th/9903238] [SPIRES].

[40] S.W. Hawking and S.F. Ross, Duality between electric and magnetic black holes, Phys. Rev. D 52 (1995) 5865 [hep-th/9504019] [SPIRES].

[41] I. Papadimitriou and K. Skenderis, Thermodynamics of asymptotically locally AdS spacetimes, JHEP 08 (2005) 004 [hep-th/0505190] [SPIRES].

[42] R. Emparan, AdS/CFT duals of topological black holes and the entropy of zero-energy states, JHEP 06 (1999) 036 [hep-th/9906040] [SPIRES].

[43] R. Brout, S. Massar, R. Parentani and P. Spindel, A primer for black hole quantum physics, Phys. Rept. 260 (1995) 329 [arXiv:0710.4345] [SPIRES].

[44] A. Wipf, Quantum fields near black holes, hep-th/9801025 [SPIRES].

[45] M. Bianchi, D.Z. Freedman and K. Skenderis, Holographic renormalization, Nucl. Phys. B 631 (2002) 159 [hep-th/0112119] [SPIRES].

[46] J. Hansen and P. Kraus, Nonlinear magnetohydrodynamics from gravity, JHEP 04 (2009) 048 [arXiv: 0811.3468] [SPIRES].

[47] M.M. Caldarelli, O.J.C. Dias and D. Klemm, Dyonic AdS black holes from magnetohydrodynamics, JHEP 03 (2009) 025 [arXiv:0812.0801] [SPIRES]. 